\title{
Wind Tunnel 'Concept of Proof' Investigations in the Development of Novel Fluid Mechanical Methodologies and Devices
}

\author{
N. Findanis and N.A. Ahmed \\ Aerospace Engineering, University of New South Wales, Sydney, \\ Australia
}

\section{Introduction}

It may be argued that the first successful flight by the Wright brothers in 1903 would not have happened had the Wright brothers not built wind tunnels and obtained aerodynamic data in a form that could be incorporated directly in the design of an actual aircraft. The wind tunnels they built at the time were of a very crude nature, but they nevertheless demonstrated conclusively their value in the aerodynamic design loop. Immediately after the historic flight, with World War I looming, a spurt in wind tunnel construction started and they were mainly built in government-funded aeronautical laboratories of Europe in countries such as Great Britain, France and Germany to achieve technical leadership in aviation between1903 and 1914.

Although, primarily designed for aerodynamic studies, the wind tunnels of today are used in a multitude of fluid mechanical investigations including aerodynamic studies. It is considered to be an essential experimental tool in fluids/aerodynamic laboratories to complement research and teaching activities around the world.

Today there are wind tunnels that are designed to cater for flow field investigations ranging from very low speeds to hypersonic speeds. The purpose of this chapter, however, is not to describe the design of these wind tunnels but to highlight the significance of wind tunnel investigations in the 'concept of proof' investigations in the development of novel fluid mechanical methodologies and devices. The materials presented here are, therefore, entirely based on the many successful and innovative works that have been carried out by N.A.Ahmed and his research teams at the aerodynamics laboratory of the University of New South Wales that spans well over a decade and supported by substantial funding from the Australian Research Council and from industry.

The chapter will start with a description of the development of novel measurement methodologies for highly three-dimensional flow fields that can be created and investigated employing a wind tunnel. In this connection the development of new methodologies for application of multi-hole pressure probes developed by the aerodynamic research group at the University of New South Wales for application in highly complex flows in wakes and in cases where reversal of flows occurs will be detailed. This will be followed by a description of the group's works where the wind tunnel investigations have featured prominently to help advance the cause of active flow control for aerodynamic efficiency of wings for aircraft 
and other fluid mechanical applications. The chapter will also demonstrate that various features and important findings from wind tunnel investigations have helped enhance the accumulation of new and significant knowledge, and validated the 'concepts of proof', that can, and also have lead to the development of practical devices such as wind driven rotating devices which are currently manufactured and marketed commercially for ventilation to ensure high inlet air quality and comfort in buildings.

\section{Concept of proof studies}

\subsection{Development of new flow measurement techniques and methodologies 2.1.1 Background information}

Investigation of fluid flows requires the development of appropriate measurement methodologies and techniques for use in wind tunnels. Wind tunnel works conducted at UNSW have resulted in the development of a mean and fluctuating pressure measurement system using plastic tubing [1], a new multi-hole pressure technique [2],[3] for pressure and velocity measurement in highly three-dimensional flows including reverse flows.

In this section, extension of the usage of multi-hole pressure measurement technique mentioned earlier to the development and validation of a novel shear stress measurement technique will be described in greater detail.

Measurement techniques for shear stress in three dimensional turbulent boundary layer flows have relied mainly on a combination of physical experimentation and subsequent empirical relationships or theoretical approaches supplemented by experimental correlation [4], [5]. The five-hole pressure measurement technique was first used in 1915. Since then, despite many attempts, it has not been possible to use this technique for flows greater than \pm $30^{0}$ angles of yaw and pitch, thereby precluding its use in many complex flow fields of practical significance. The new technique developed at UNSW showed that it can be used in flows of angularity as high as $\pm 85^{\circ}$ angles of yaw and pitch by overcoming the singularity problem associated with previous existing techniques.

The initial work by Lien and Ahmed [6] involved the development of a novel skin friction measurement technique in two dimensional flow using multi hole pressure probe. This has been followed by the experimental study using wind tunnels is to further extend its application to measurements of skin friction in three-dimensional turbulent boundary layer [7] by measuring the shear stress angle more accurately.

It should be mentioned that the major difficulty associated with the measurement of shear stress angle is that its magnitude is small. The case is further complicated for three dimensional boundary layer where the presence of the pressure gradient normal to the free stream flow direction due to curvature forces the fluid particle in the boundary layer tend to accelerate in the normal direction. Due to their lower velocity, the fluid particles must travel on trajectories of smaller radius of curvature than the free stream flow in order to balance to pressure field imposed on them by the flow above. Due to the extra dimension caused by the skewing of the flow, the measurement of shear stress angle becomes important.

Skewing of the flow in three dimensional boundary layer flows which refers to the boundary layer velocity vectors not parallel to the main flow velocity which can be described in a streamline coordinate system is shown in Fig. 1. This skewing is caused by streamline divergence or convergence which the boundary layer velocity vectors are collateral with the main flow velocity vector at a point while the secondary flows are not parallel to the main flow velocity vector. 


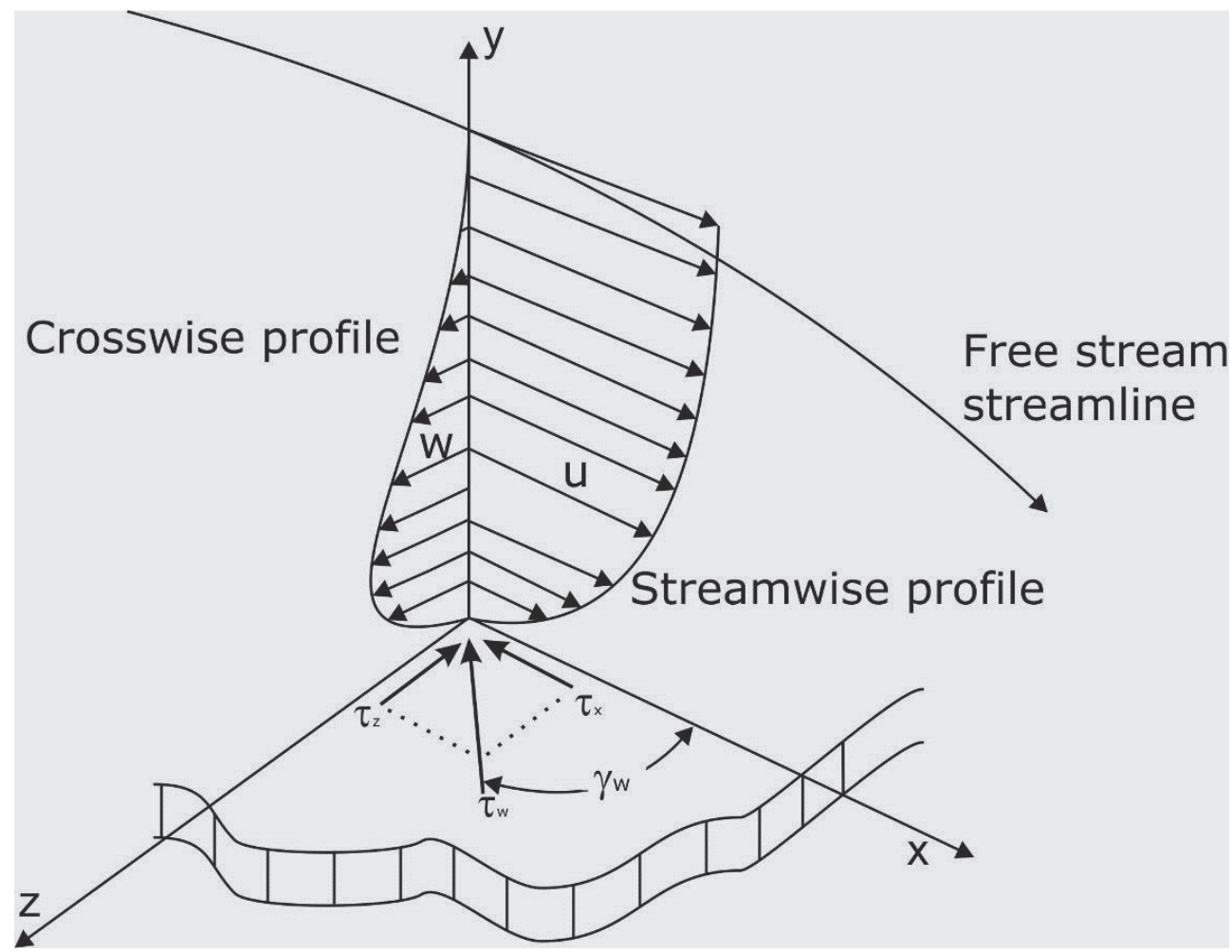

Fig. 1. Schematic of velocity profiles in three dimensional turbulent boundary layer

Several flow measurements in three dimensional flow field have been carried out using pressure probes. The measured flow parameters are usually the total, static pressure as well as the flow angle at the measurement location and these have been carried out by Pitot tubes and yaw sensing tubes or combination of them as two- or three-hole pressure probes in a nulling mode where the probe is rotated until zero pressure difference is obtained across the two side slanted tubes at teach profile point. For example, Johnston [8], Elsenaar and Boelsma [9], and Chandrasekhara et al [10] use three-hole pressure probe soldered side by side and cut off at the tip with a apex angle in three dimensional flows. The use of pressure probes in a non nulling mode can also be used to determine the direction of the flow within the boundary layer where the pressure difference is pre calibrated in a known flow [11].

One major difficulty of insertion of a pressure probe into a pressure driven flow field is that it introduces error in the measurement of flow parameters as some extra flow interactions of the stream lines and the probe are induced. However studies by Vagt [11] also points out that such effects are not severe in shear driven three dimensional boundary layers or where the surface curvature is weak. The sensitivity of pressure probes technique depends largely on the probe geometry as well as the accuracy of the manometer. It was demonstrated that pressure probes are capable of measuring flow angles in turbulent boundary layer accurately within $\pm 0.1^{0}$ with suitable choice of probe geometry and the assist of highly sensitive pressure transducer or micromanometer [11],[12]. These studies lead to a 
conclusion that with careful selection of pressure probes, they can be used in accurate determination of flow quantities such as flow angles and velocity profiles even in three dimensional turbulent boundary layer.

Hence, the present study is an effort to extend the use of multi hole pressure probe for measurements of skin friction and shear stress angles in three-dimensional turbulent boundary layer.

\subsubsection{Experiment}

A test rig, Fig, 2, was constructed at the aerodynamics laboratory of the University of New South Wales. The experiments were conducted in a $76 \mathrm{~mm}$ diameter open return, $0.2 \%$ turbulence intensity open test section wind tunnel of the Aerodynamics Laboratory of the University of New South Wales.

Uniform and parallel freestream flow is produced by an open jet wind tunnel and passes through the entry duce. The top and bottom walls are kept far apart to ensure the boundary layer on both walls will not interfere with each other. The flow is forced to turn sideways due to the effect of pressure gradient introduced by the back wall. A detailed description of the test apparatus is given in Fig. 2a.

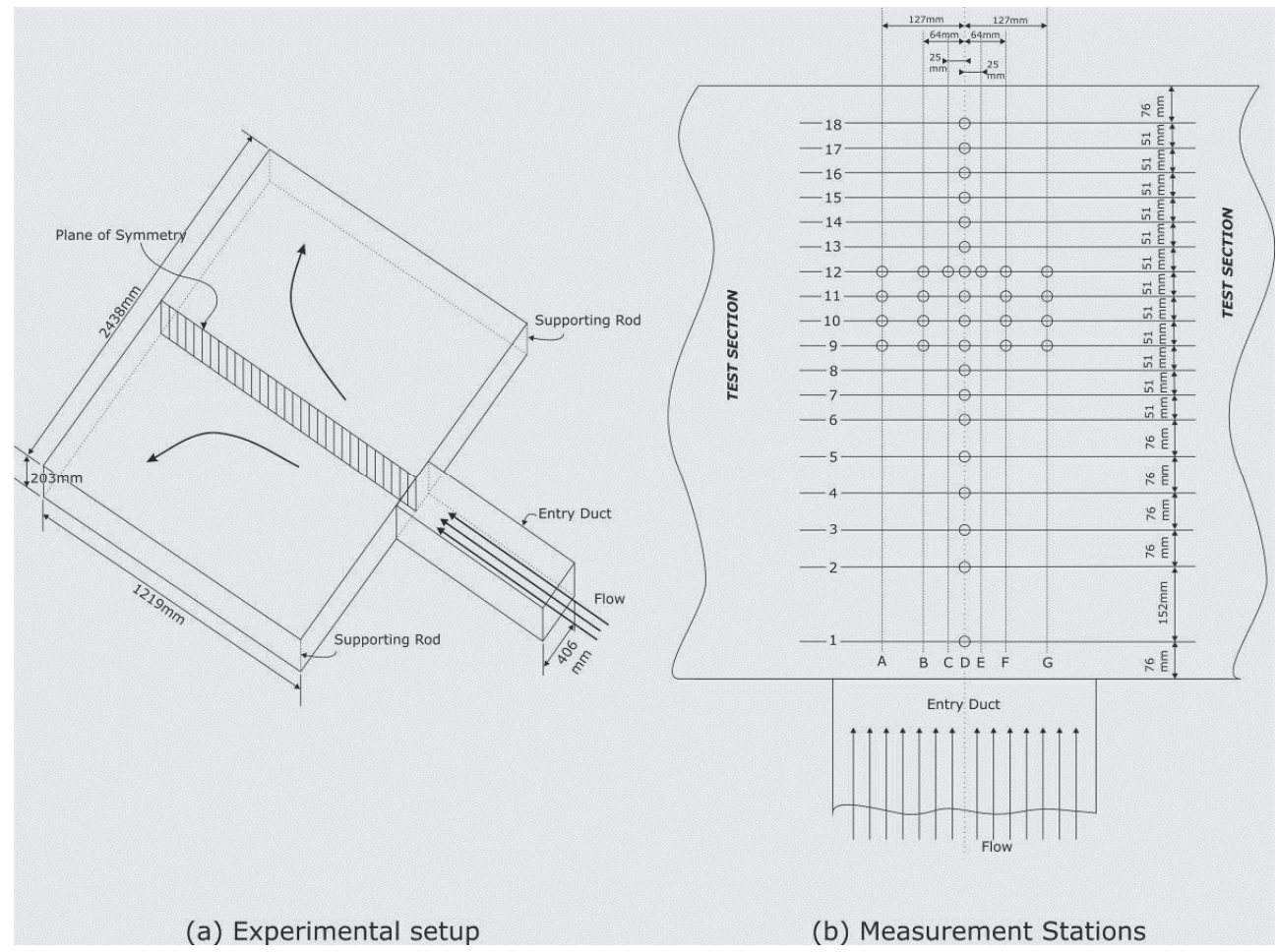

Fig. 2. (a) Experimental setup; (b) measurement stations 
The reference speed of $31 \mathrm{~m} / \mathrm{s}$ was held constant for all tests which is derived from the Pitot-static probe reading located at the entry of the duct. The air used in the test section was nominally at room temperature and atmospheric pressure.

The measurement stations are shown in Fig. $2 \mathrm{~b}$. The velocity profiles at each measurement station are determined by traversing a five hole pressure probe of $2.3 \mathrm{~mm}$ diameter at different heights across the boundary layer. The upstream profiles are also measured at the plane of symmetry represented by Row $\mathrm{D}$ to ensure uniform two-dimensional condition is valid for the main entry flow.

A traversing gear, allows movement in both horizontal $(\mathrm{X})$ and vertical $(\mathrm{Y})$ direction with an accuracy of $\pm 0.01 \mathrm{~mm}$ was used in the investigation. A bank of Honeywell transducers and a FCO510 micro-manometer from Furness Controls, Ltd, was used to obtain the pressure data from the five-hole pressure probe and the freestream velocity from the Pitot-static probe, respectively. The manometer and the pressure transducers provide measurements ranging from $\pm 2000 \mathrm{~Pa}$ with accuracy up to $0.1 \mathrm{~Pa}$. Through a set of calibration curves similar to these mentioned in Pisasale and Ahmed [13] the value of (Pt - Ps) as well as the yaw and pitch angles were found and the velocity of the flow is determined.

The experimental program was accomplished in two phases. The first phase defines the mean flow field where mean velocity profile, yaw angle and pitch angle were measured at different measuring locations. The second phase is to derive the shear stress components, including the shear stress angle from the pressure readings of each holes.

\subsubsection{Results and discussions}

\subsubsection{Shear stress angle}

For skewed boundary layer, the shear stress angle near the wall can be determined from its velocity profile, which is the ratio of $w$ and $u$. The two velocity components ( $u$ and $w$ ) from the five pressure probe technique at a point can be can be expressed as follows:

$$
\begin{aligned}
& u=\cos \alpha \cos \beta \\
& w=\sin \alpha
\end{aligned}
$$

Hence:

$$
\begin{aligned}
\gamma_{w} & =\tan ^{-1}\left(\frac{w}{u}\right)_{\text {near wall }} \\
& =\tan ^{-1}(\tan \alpha \cos \beta)
\end{aligned}
$$

The yaw angle measured at the plane of symmetry is presented at Fig. 3. Since the flow along the plane of symmetry will not turn before the separation zone, the shear stress angles are expected to be around zero values at this plane. The results showed that the measured shear stress angles using five hole pressure probe are closed to zero values at the plane of symmetry.

The measured shear stress angle using the multi hole pressure probe method also showed better agreement with the angles derived from the velocity profiles than those measured by Johnston for this plane. 


\subsubsection{Skin friction measurements}

After the examination of shear stress at each measurement station, the skin friction values obtained from multi hole pressure probe technique by Lien and Ahmed [7] were compared again with calculated values using momentum integral equations in its three dimensional form.

The various momentum integrals along measurement plane D are presented in Fig. 4 which the measured skin friction using multi hole pressure probe is plotted in Fig. 5. The skin friction values measured by five hole pressure probe were found to be in good agreement with Johnston's experimental data and momentum integral technique.

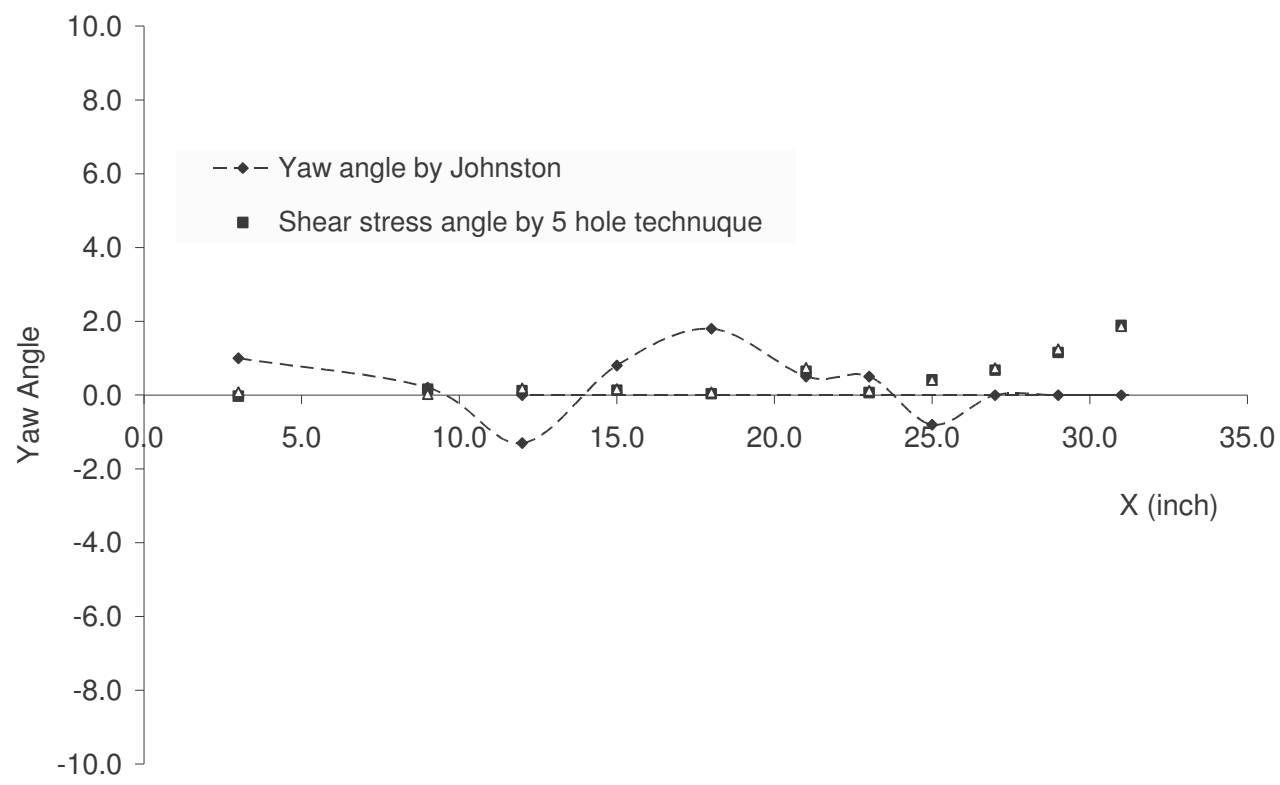

Fig. 3. Measured shear stress angle along the plane of symmetry 


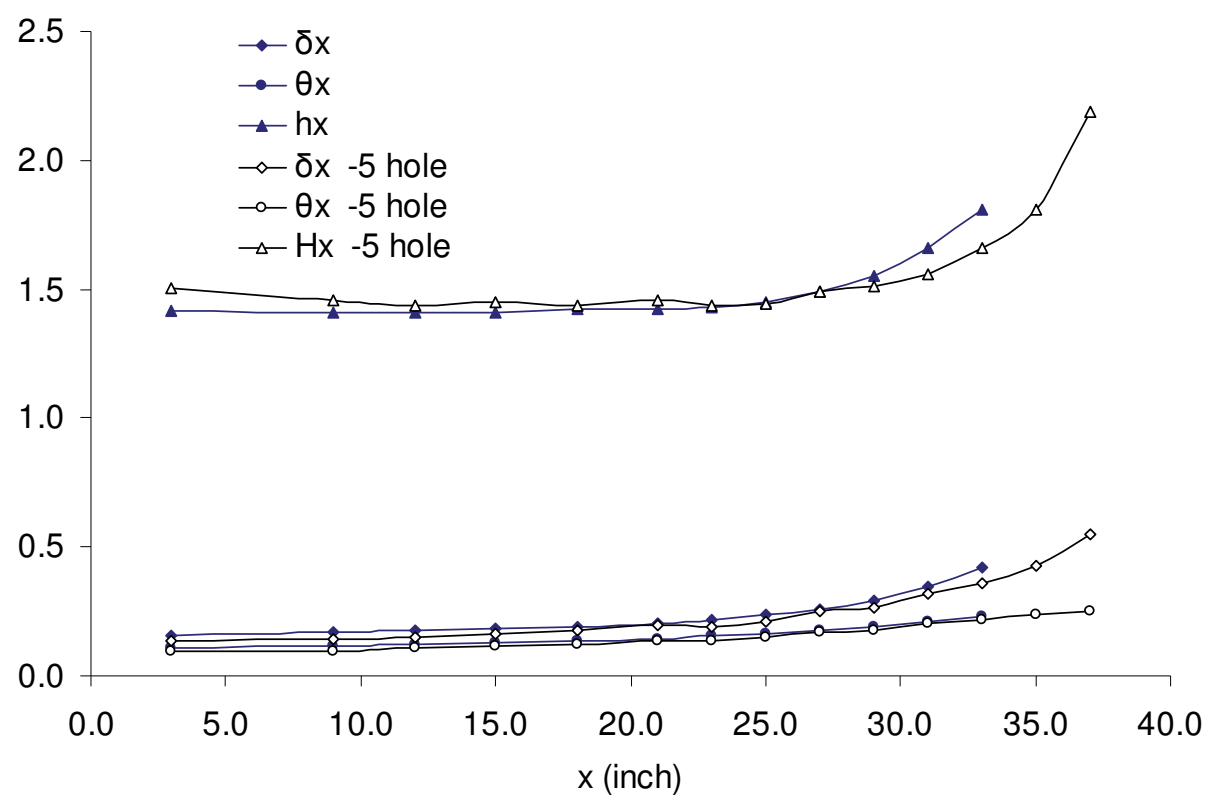

Fig. 4. Various momentum integral parameters along the plane of symmetry

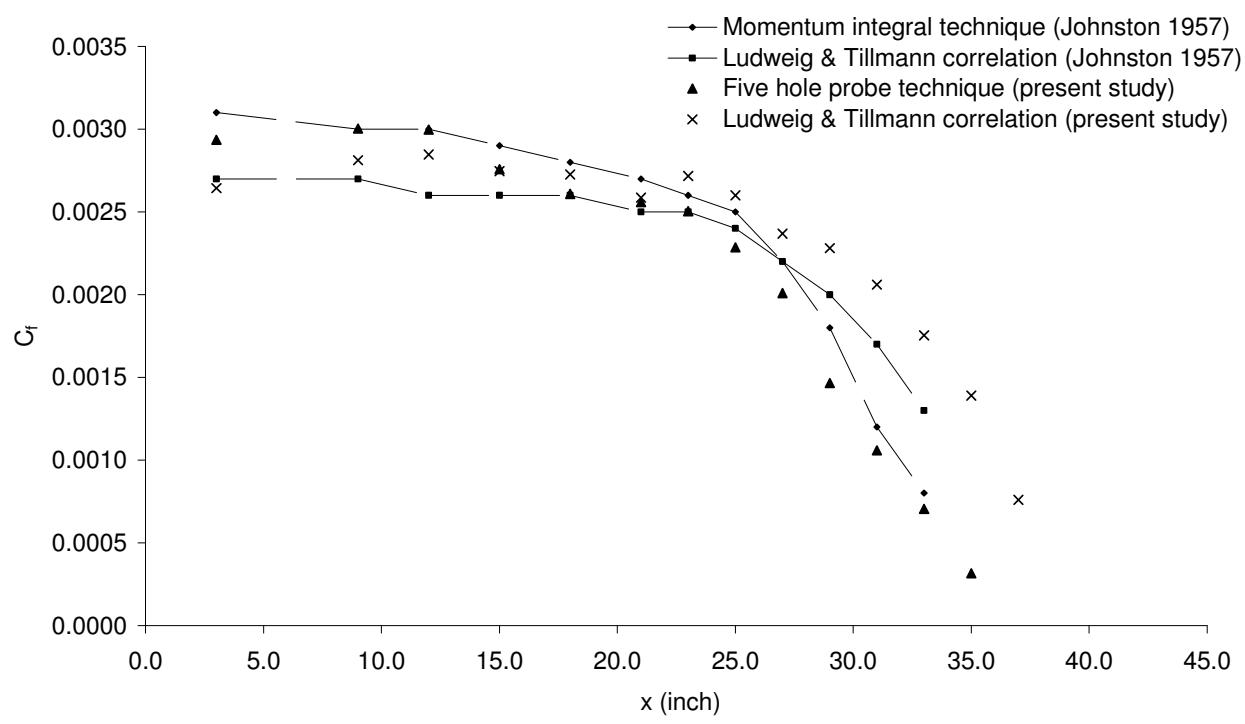

Fig. 5. Skin friction along the plane of symmetry 


\subsubsection{Conclusions}

A method of determining local skin friction developed by Lien and Ahmed (2006) using multi-hole pressure probe has been presented in this study. The method is based on wall similarity law and the utilization of the logarithmic law of the wall.

Wind tunnel tests were conducted to obtain skin friction values in three dimensional turbulent flows. It is worth noting that using the present method, the skin friction is deduced from $\Delta \mathrm{P}$, that is the method essentially determines a vector quantity from scalar information. The ability of non-nulling multi-hole pressure probe, however to determine $a$ and $\beta$ values means that the directional aspect of the skin friction may be easily determined. The present study suggests that the skin friction value as well as shear stress angle can be determined from multi-hole pressure probe reading when the probe is placed within the wall region. This is very promising and points to the possibility of extending the technique to more complex three dimensional flows as well where non- zero values of $a$ and $\beta$ occur. Since the multi hole pressure probe method is based on similarity principle where the probe diameter is used as a characteristic parameter, it is expected that the method would be equally effective with a different probe geometry provided it is small enough to be submerged in the boundary layer and that its calibration coefficient can be accurately determined.

\subsection{Investigation of synthetic jet for active flow control \\ 2.2.1 Background information}

Substantial experimental work with synthetic jets in various flow regimes and in different types of flow i.e. laminar and turbulent flows, has been performed over recent years moreover there has been an escalation as they continue to draw the attention of researchers in fluids. Some applications of the synthetic jet include flow separation control and turbulence, control of thrust vectoring, augmentation of heat transfer and mixing, and microfluidics. Specifically synthetic jets are proving to be a beneficial device in terms of active flow control in applied aerodynamics. This has been demonstrated with the use of synthetic jet in subsonic crossflows in which typically the synthetic jet emanates from the surface of a bluff or streamlined body reducing the overall drag and wake region as evidenced in the works of Glezer and Amitay [13]. Interestingly, synthetic jets improve the flow by imparting a net momentum transfer to the flow without a net mass addition thereby delaying flow separation and reattachment this altered flow also generates a separation bubble above the surface of the body. The flow structure of synthetic jets consists of a time-periodic formation of vortex pairs that eventually progress into transition to turbulence and the monotonic decrease in their stream wise velocity that ultimately leads to their dissolution.

Although several studies have been conducted on synthetic jet in a crossflow such as the flow over a circular cylinder [13], [14], [15], there is a noticeable lack of studies on true threedimensional bodies with synthetic jet [16]. The flow over the cylinder can be described with two independent variables and as such is termed axis symmetric. It is a degenerate threedimensional flow that exhibits certain three-dimensional effects and as such it is not really a two-dimensional flow and not fully three-dimensional either. A common phenomenon to all three-dimensional flows is the three-dimensional relieving effect that is present in flow over a sphere but not that of a cylinder. A greater understanding of the synthetic jet in threedimensional flows is essential to enable successful inception in various engineering applications especially in the aerospace industry. 
Synthetic jets may be applied either in a distributed manner or from localised sources also oscillatory streaming motions can be induced in the fluid acoustically through the transmission of sound or 'acoustic streaming' [16], [17]. A case in hand of acoustic excitation is the work of Kim and Durbin [18] of a uniform acoustic field applied symmetrically from a distance to the airflow interacting with the sphere in the Reynolds number range of $500<\operatorname{Re}$ $<60,000$. The results indicated that when the acoustic excitation was near to the natural instability of the flow a decrease in the wake size occurred as a consequence of the separation point being delayed and pushed further downstream towards the wake region. This created a rapidly thickening but broader and shorter recirculation zone from the inwardly directed shear layer. This also produced a lower base pressure behind the sphere and possibly in the near wake region with a higher velocity in the recirculation zone as a result of a reduced size of the separation bubble. Since the forcing was being applied away from the body, an increase in the turbulence level with a consequent increase in the base pressure over the body was produced which then resulted in higher drag.

Additionally experimental investigations carried out by Jeon et al. [16] on a sphere with a uniform acoustic field emanating from a slot located at an angle of $76^{\circ}$ from the fore stagnation point but equally distributed circumferentially around the sphere, showed that for forcing frequencies equal to or greater than the critical frequency, St $=2.85$ or $190 \mathrm{~Hz}$, there was a reduction in drag of nearly $50 \%$. This forcing frequency corresponded to the boundary-layer instability frequency that was the high shear-layer instability frequency. . The boundary layer instability frequency falls in between the low wake instability frequency (vortex shedding) and the high shear layer instability frequency which at this Reynolds number are St $=0.18$ and St $=10$ respectively. The works of Kim and Durbin [18] as described above showed that the reverse flow region became stronger and that the total drag was increased with acoustic forcing. Jeong et al. [16] has suggested that the mechanism for reducing drag at the critical frequency could be due to the corresponding high frequency boundary-layer instability.

Further to this, synthetic jet can be applied in a distributed manner. The work by Glezer and Amitay [15] with a synthetic jet placed locally on one side of a cylinder showed a reduction in surface pressure both upstream and downstream of the forcing location and induction of a local separation bubble above the location of the synthetic jet and the "virtual aeroshaping" [19] of the body that was responsible for the substantial reduction in drag values. It is not well established as to when or under what conditions these bubbles will form particularly in three-dimensional flows with the synthetic jet impinging on the cross-flow and forming a recirculation zone.

The present study was therefore conducted to contribute to the need for three-dimensional experimental work in an effort to gain a further understanding of the three-dimensional effects encountered when applying a synthetic jet on a three-dimensional body in a crossflow. A parallel purpose of this present study is to investigate the validity of using a five hole probe to measure the total and static pressures at a point and then indirectly calculate the velocities in a highly three-dimensional wake. Since the flow over the sphere is a true three-dimensional flow it also being a simple and symmetrical three-dimensional bluff body this became a suitable choice with which to perform our experiments. The present study presents a rather straightforward and effective way to examine the threedimensional behavior of the bluff body in terms of boundary layer growth, skin friction, separation of flow and pressure drag changes in three-dimensions under the influence of a localized synthetic jet. Designing the experiment in this fashion provides the distinct 
advantage of being able describe the influence of the synthetic jet at a particular angular location relative to the free stream.

It is interesting to note that the maximum Reynolds number at which Kim and Durbin [18] and Jeong et al. [16] conducted their experiments form approximately the two limits of the plateau like region on the $C D$ curve for a sphere [20] where the drag practically remains unchanged with changes in the Reynolds number. It appears that the effect of a localised synthetic jet on the flow over a sphere at a Reynolds number that lies within those limits would be a suitable choice and the present study was, therefore, conducted at a Reynolds number of $5 \times 10^{4}$.

\subsubsection{Experiment}

The experimental work was conducted using an $18^{\prime \prime} \times 18^{\prime \prime}$ open circuit, closed test section, NPL type wind tunnel at UNSW aerodynamics laboratory [21], [22]. The wind tunnel was operated at an air speed of $10 \mathrm{~ms}^{-1}$. The bluff body used for the experiments was an aluminium sphere of $80 \mathrm{~mm}$ diameter and supported horizontally by a rod attached to one side of the sphere. Using the method of Rae and Pope [23], the blockage ratio was calculated to be $2.8 \%$. Additional velocity profiles obtained using hot-wire anemometry on the centreline plane of the sphere showed little or no difference from the free stream velocity profile confirming that tunnel blockage was negligible. The sphere was designed with a synthetic jet orifice (labelled SJ in Fig. 6) of $1 \mathrm{~mm}$ diameter and oriented at a $45^{\circ}$ tangent to the normal of the sphere surface and seven pressure ports or tapping points (numbered 1, 2, $3,4,5,6,7)$ on the centreline axis of the sphere (Fig. 6). The sphere was rotated to each predetermined angular position after which the wake survey measurements were taken.

The synthetic jet actuator was composed of a modified compressor and a $12 \mathrm{~V}$ power supply. The compressor piston was fitted with a one-way valve that was modified to cause suction and blowing as it reciprocated instead of constant compression of the air. The compressor line was connected to the synthetic jet orifice on the sphere using silicon tubing. The sphere support was fitted against a fixed protractor to measure the angle of rotation.

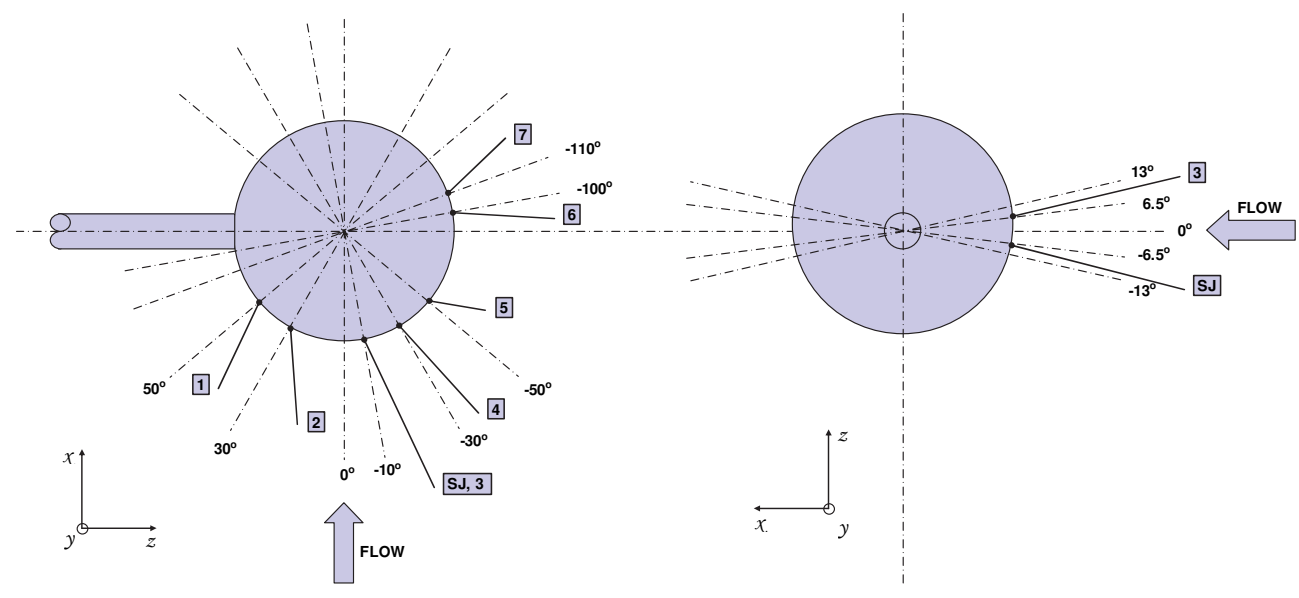

Fig. 6. Schematic diagrams of sphere: Plan view (left), Elevation view (right) 
The velocity of the synthetic jet was measured using a Dantec Streamline CTA Anemometer system with a 55P14 probe. The probe was calibrated using the Dantec Flow Calibration System. The voltage supplied to the motor was through a DC power supply unit to generate the desired frequency with a range of $10 \mathrm{~Hz}$ up to $75 \mathrm{~Hz}$. The frequency obtained from hot-wire measurement was cross-checked against the rotational speed of the motor using a laser tachometer. Since the hot-wire measurement was indifferent to flow direction and the two extremes, that is blowing and suction, were 180 degrees out of phase, the peaks are both positive for suction and blowing. The maximum velocity measured was about $35 \mathrm{~ms}^{-1}$, and its rms value was $15 \mathrm{~ms}^{-1}$. The actuation frequency was $\mathrm{f}=60 \mathrm{~Hz}$ that corresponded to a Strouhal number of $S t=0.53$. This value was chosen to avoid correlation with the low wake instability frequency (vortex shedding frequency) and the high instability frequency (separating shear layer instability frequency) which are at St $=0.19$ and St $=6$ respectively at the present Reynolds number. Rather than examining the effect of the synthetic jet as targeted towards flow instabilities this paper focuses on the mechanism of momentum transfer as a means to control the flow over the sphere and thereby improve aerodynamic performance through a reduction in drag. Work on a two-dimensional body [17] has shown an improvement in the delay of separation for a Strouhal number of 2.5 for a circular cylinder. Whether similar improvement of the flow over three-dimensional flow can be obtained with the application of the localised synthetic jet was, therefore, a major motivation of this project.

\subsubsection{Results and discussions}

\subsubsection{Qualitative: Flow visualization}

The flow visualisation using the tufts was intended to provide some qualitative clarity regarding the improvement in flow due to the activation of the synthetic jet. The synthetic jet was orientated at an angle of $80^{\circ}$ which is approximately the location of separation for a standard sphere Schlichting [20]. Figs. 7a to $7 \mathrm{~d}$ are flow visualisation results observed at wind speed of $10 \mathrm{~ms}^{-1}$. Figs. $7 \mathrm{a}$ and $7 \mathrm{~b}$ show that the flow is reversing as evidenced by the tufts tending to curl back onto the screen and flutter back towards the sphere. In Figs 7c and $7 \mathrm{~d}$, also at an airspeed of $10 \mathrm{~m} / \mathrm{s}$, a different picture emerges and the tufts can be seen pointing back in the streamwise flow direction. This shows a significant straightening of the flow field in the wake of the sphere.

Fig. $8 \mathrm{c}$ shows the flow pattern of the tufts at $10 \mathrm{~ms}^{-1}$ with the synthetic jet activated at $50 \mathrm{~mm}$ from the sphere. This flow pattern is similar to a wake flow pattern of $20 \mathrm{~ms}^{-1}$ at $40 \mathrm{~mm}$ from the sphere surface as shown in Figs. $8 \mathrm{a}$ and Fig. $8 \mathrm{~b}$ which has the tufts at $80 \mathrm{~mm}$ from the sphere surface without the synthetic jet activated. These figures suggest that the wake of the sphere has been shortened by approximately $30 \mathrm{~mm}$ when synthetic jet is activated.

\subsubsection{Quantitative: Wake flow field measurement}

The wake flow field was defined in terms of velocity information obtained using pressure measurements from the five-hole probe and then plotted as 3-dimensional velocity vectors in Figs. 9(a) to 9(d) using the Matlab software program.

The difference in velocity field when the synthetic jet is activated can be seen by the increase in vector magnitude and a straightening of the vectors. The decrease in wake region also provides evidence of the effectiveness of the synthetic jet. These 3-dimensional vector plots correspond to the results of the flow visualisation where we see in both types of results a shortening of the wake region by approximately $30 \mathrm{~mm}$. 


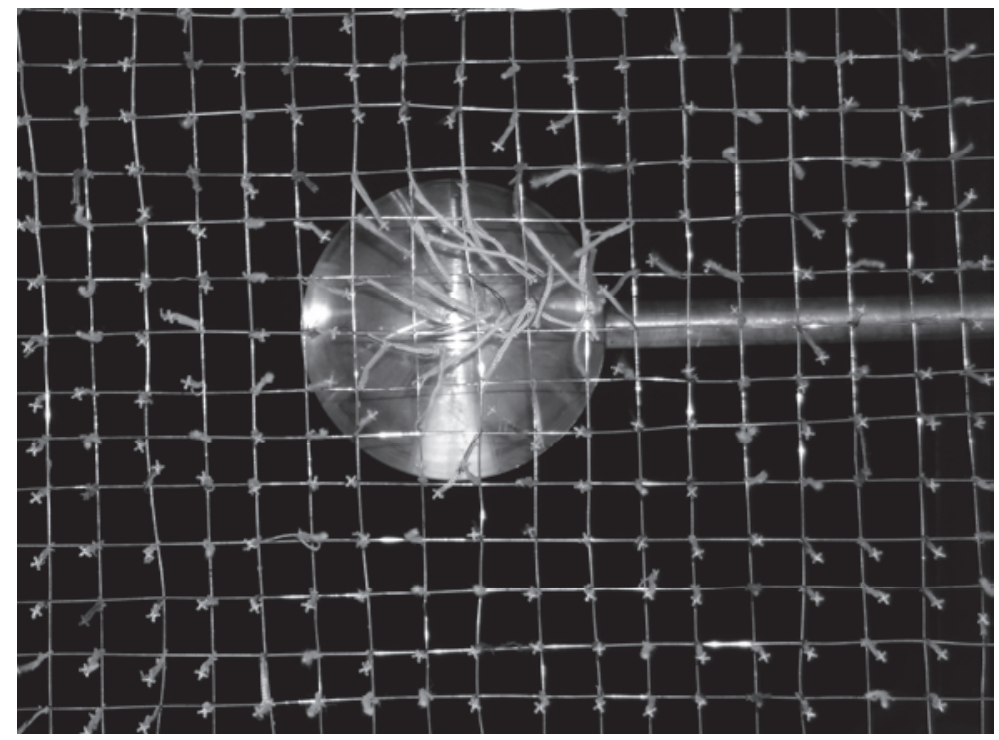

(a)

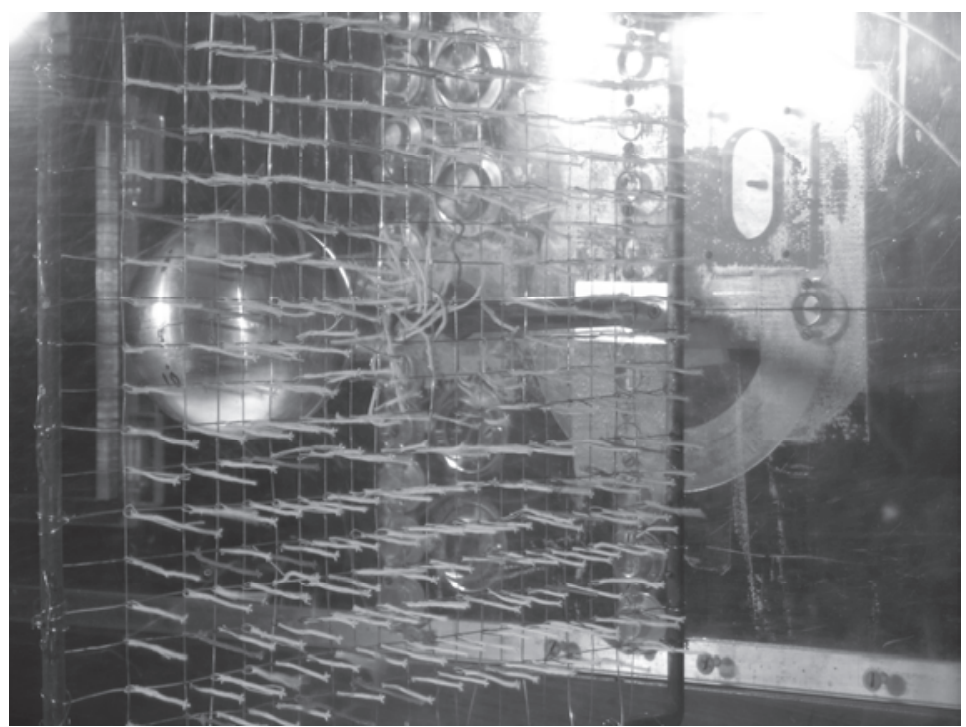

(b) 


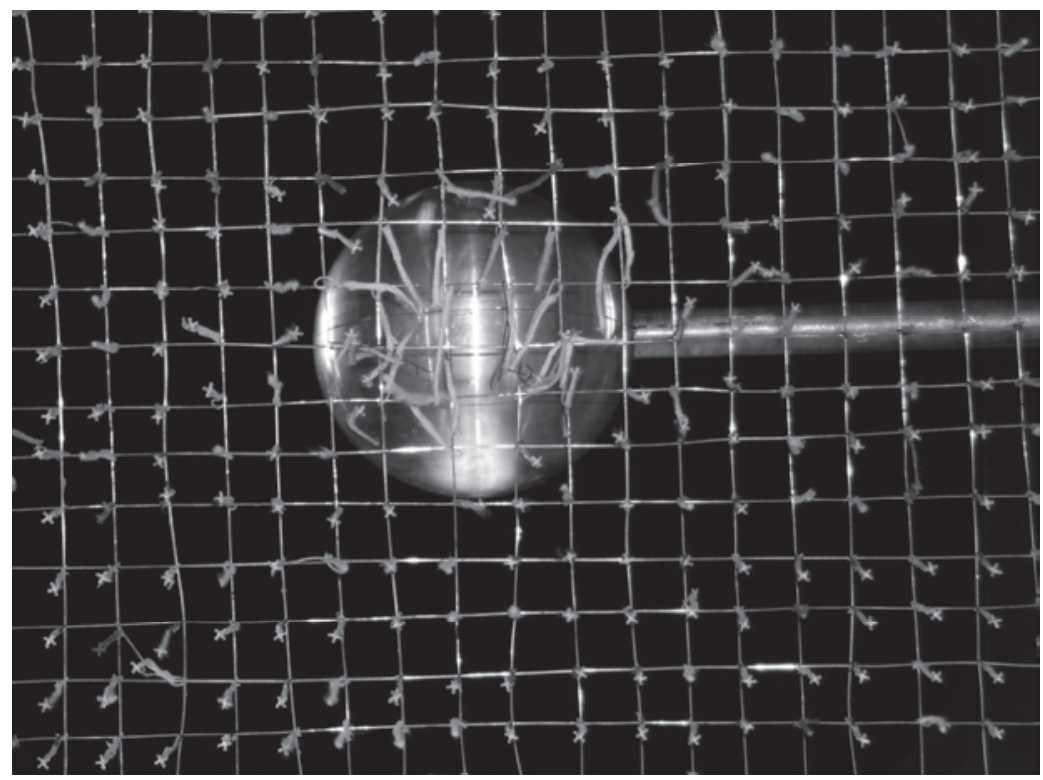

(c)

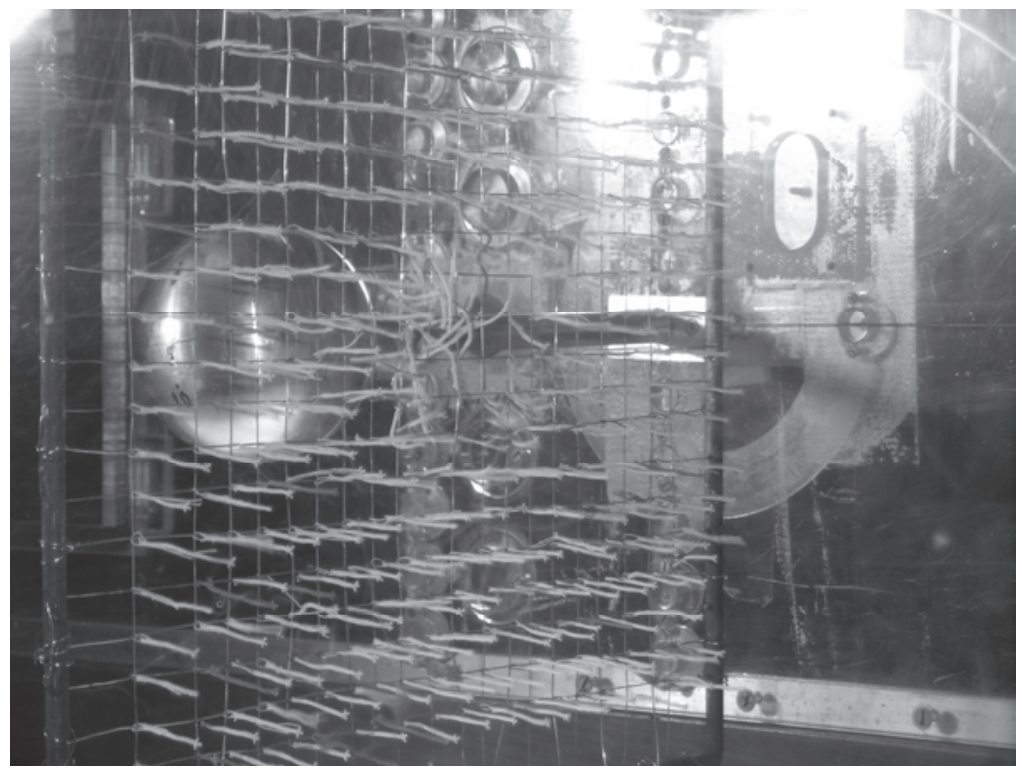

(d)

Fig. 7. (a) $10 \mathrm{~m} / \mathrm{s}$ without synthetic jet. Tuft screen $40 \mathrm{~mm}$ off-set.; (b) side view of (a) ; (c) $10 \mathrm{~m} / \mathrm{s}$ with synthetic jet. Tuft screen $40 \mathrm{~mm}$ offset. (d) side view of (c) 


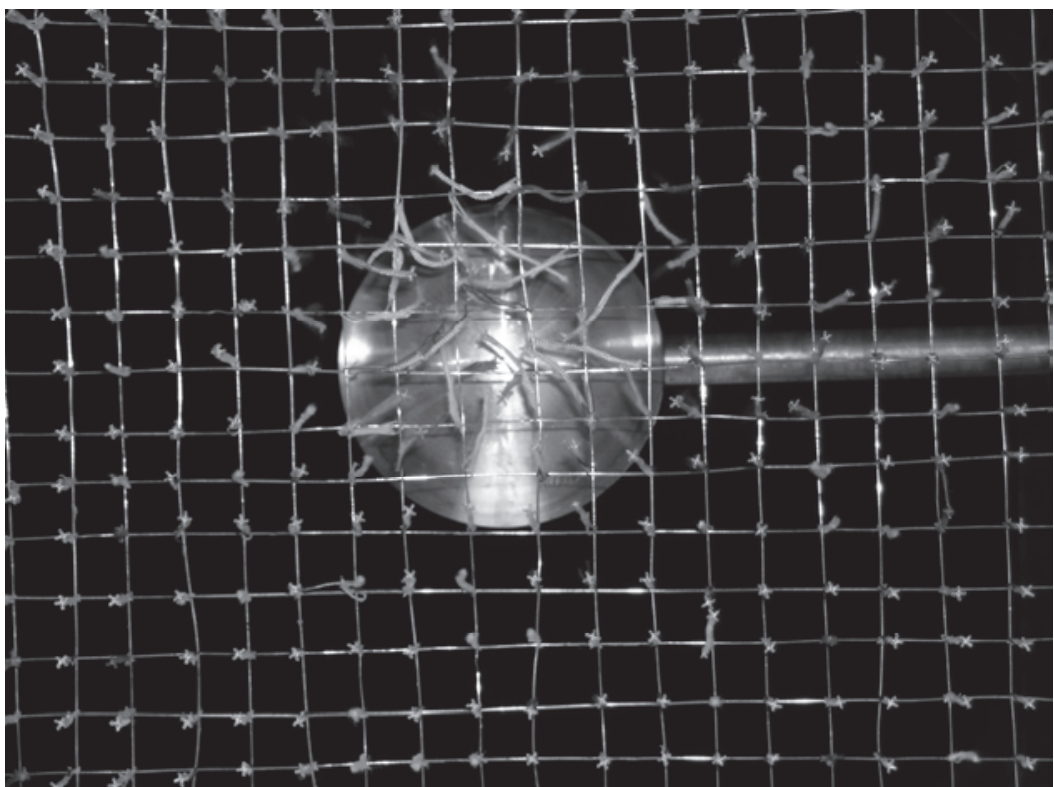

(a)

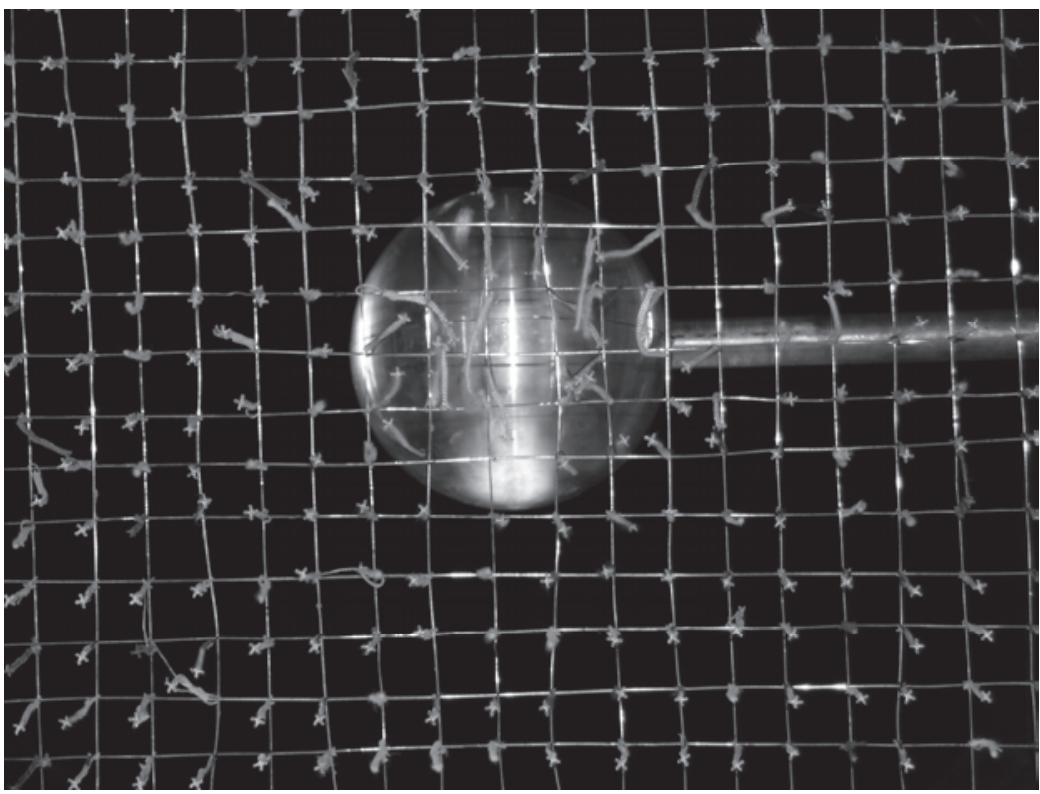

(b)

Fig. 8 . (a) $20 \mathrm{~m} / \mathrm{s}$ with synthetic jet. Tuft screen at $40 \mathrm{~mm}$ offset; (b) $10 \mathrm{~m} / \mathrm{s}$ without synthetic jet. Tuft screen at $80 \mathrm{~mm}$ offset; (c). $10 \mathrm{~m} / \mathrm{s}$ without synthetic jet. Tuft screen at $50 \mathrm{~mm}$ offset. 


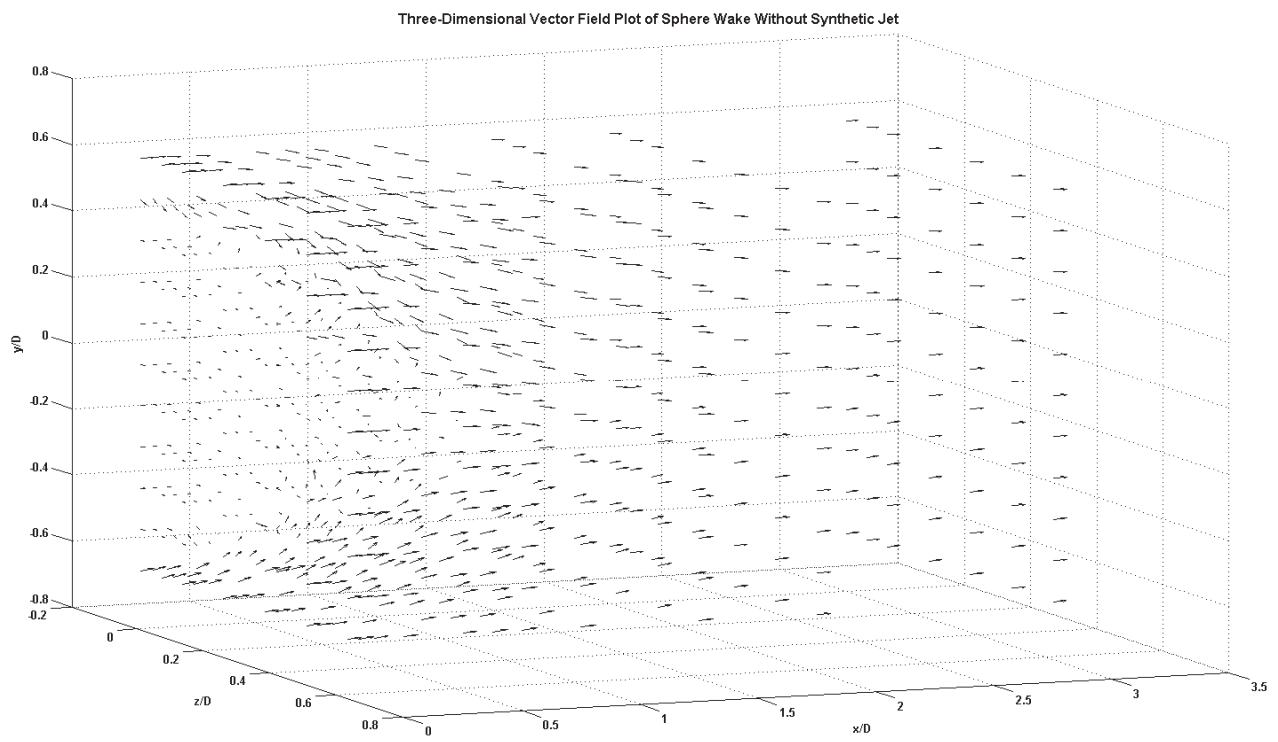

(a)

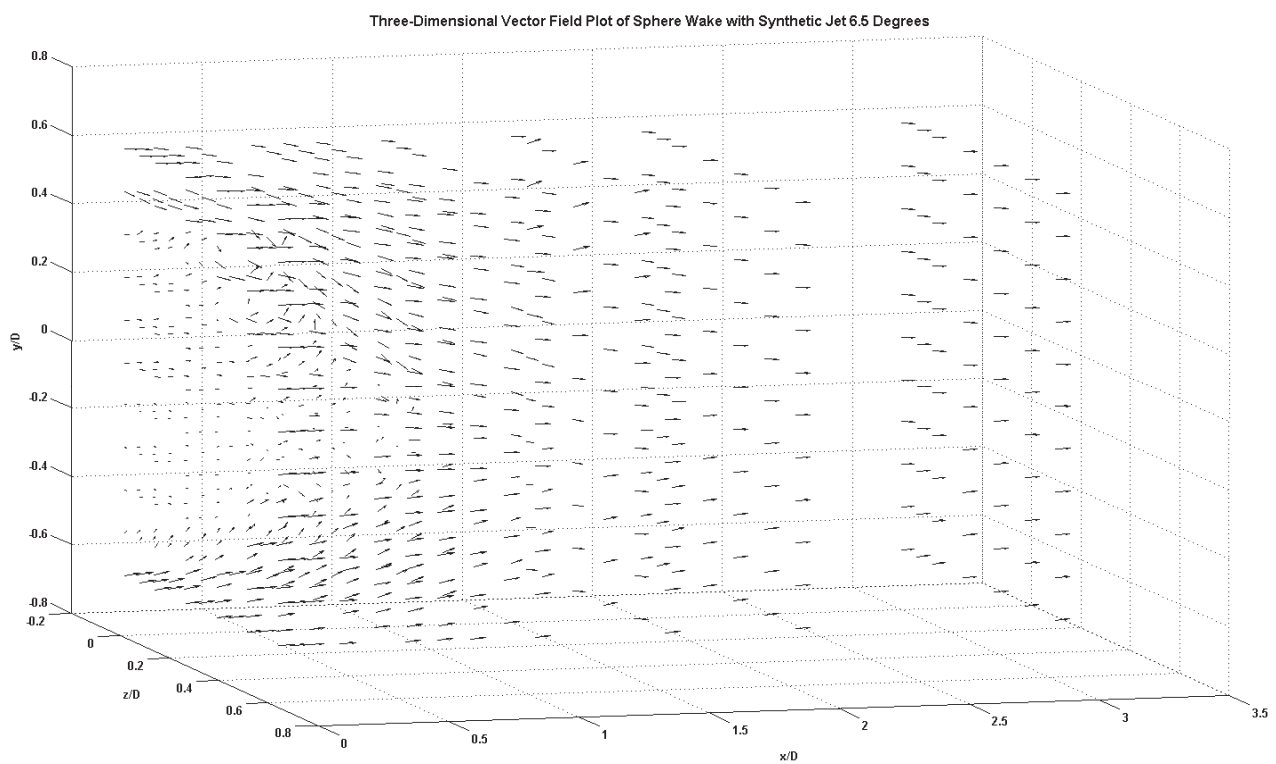

(b) 


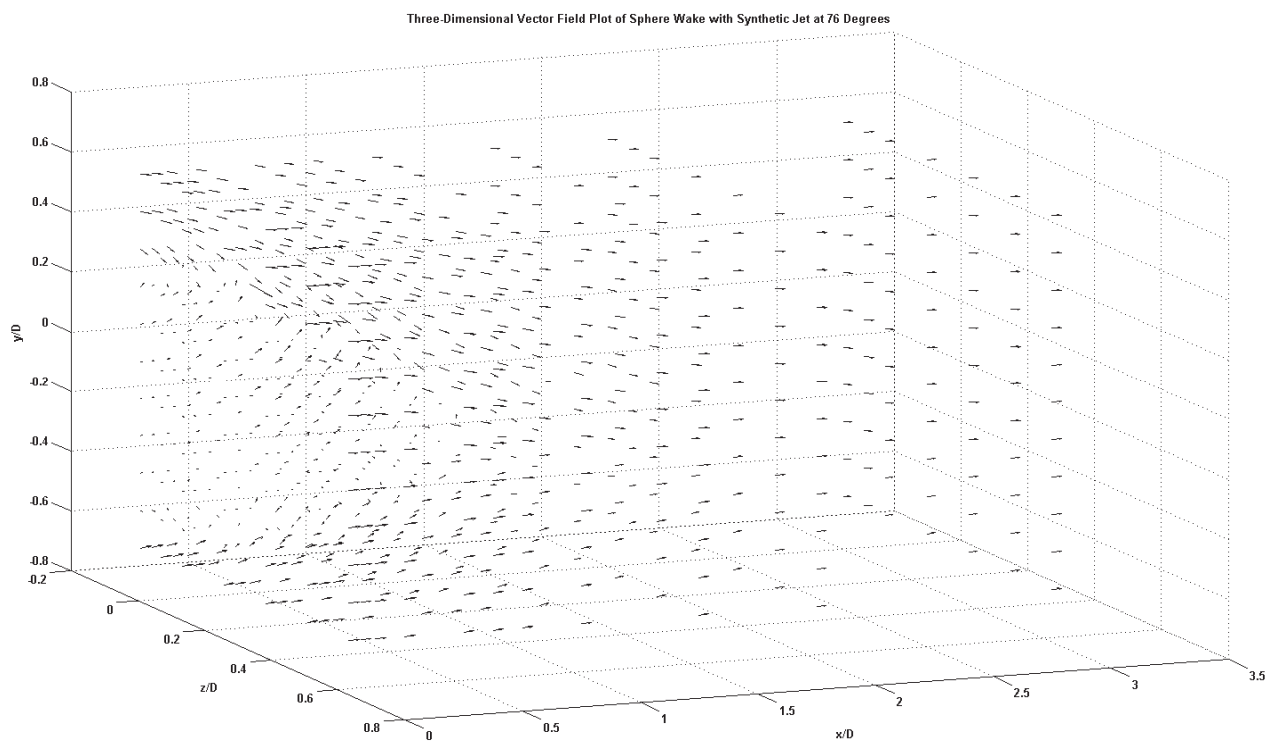

(c)

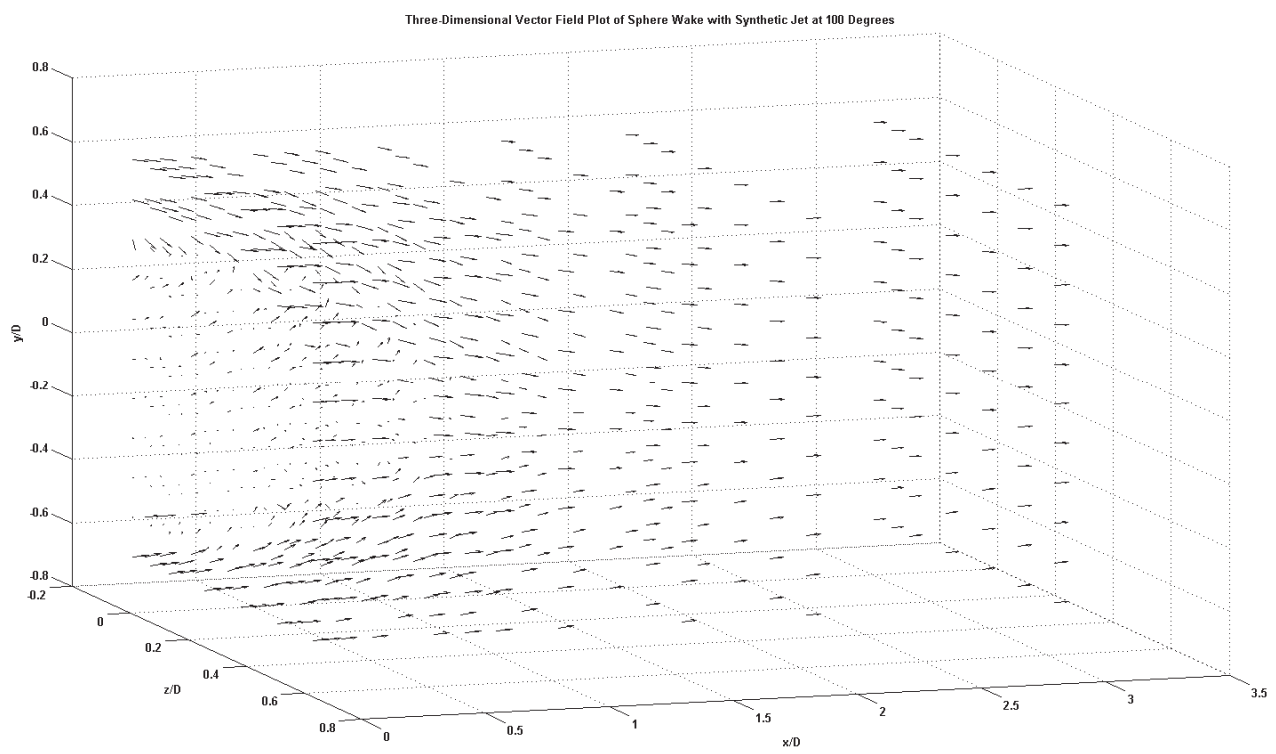

(d)

Fig. 9. (a). 3-D Vector Field Plot of Sphere Wake Without Synthetic Jet ; (b). 3-D Vector Field Plot of Sphere Wake with Synthetic Jet at 6.5 Deg; (c). 3D Vector Field Plot of Sphere Wake with Synthetic Jet at 76 Deg; (d). Three-Dimensional Vector Field Plot of Sphere Wake with Synthetic Jet at 100 Degrees 
The underlying motivation is to understand the behaviour of fluid flow with a change in the flow velocity as the Reynolds Number varies with the influence of the localised synthetic jet. In the present work we show that global changes occur to the wake of sphere at the much lower angle of incidence of the synthetic jet location of $6.5^{\circ}$ above the stagnation point and a higher momentum coefficient of $2.8 \times 10^{-3}$. Furthermore the works of Glezer and Amitay [17] show that for an angle of incidence of $60^{\circ}$ that the velocity profile is reduced, that is the velocity defect is reduced, and thus this shows that the drag has been reduced. This also occurs at the lower angle of $6.5^{\circ}$ for the sphere although with a lesser effect at this angle as would be expected since at this angle the effects of the synthetic jet have been much reduced since the vortices emanating from the synthetic jet orifice have a greater distance to travel inside the boundary-layer and thus the effects of skin friction would also dissipate the energy of the added effects of the synthetic jet. Whereas when we place the synthetic jet at an angle of $76^{\circ}$ the added energy of the synthetic jet can affect the shear layers of the flow and the thus have a straightening effect on the flow over the sphere. Thus the velocity defect in the wake region is much reduced and thus the drag which is due to pressure drag is reduced also. Since the majority of the drag for the sphere is due to the pressure drag the synthetic jet at $76^{\circ}$ produces a greater reduction on drag than when the synthetic jet is at $6.5^{\circ}$ which produces a reduction in skin friction drag as well some reduction although a lot less in form or pressure drag than when the synthetic jet is angled at $76^{\circ}$.

\subsubsection{Conclusions}

This flow study has shown a localised synthetic jet is an effective tool for aero-shaping typical 3-dimensional bluff bodies. The change in the coefficient of pressure is effectible over the surface of the sphere by placing the synthetic jet at a location upstream or downstream of the separation point as was the case with the cylinder experiments conducted by Glezer and Amitay[7]. The synthetic jet influence decreases as the distance form the centre of the sphere increases.

The wake region of the sphere was decreased through the use of the synthetic jet at both angular locations. The synthetic jet has the effect of tripping the flow and preventing recirculation or reversal of flow in the wake of the sphere. The wake region was seen to decrease by approximately $30 \mathrm{~mm}$ at an airspeed of $10 \mathrm{~m} / \mathrm{s}$. The 3 -dimensional velocity field with the synthetic jet operating indicates an increase in the streamwise component. Indicating that the possible flow reversal has been eliminated and vorticity has been lessened.

The localised synthetic jet with a cross flow Reynolds number of $5.1 \times 10^{4}$ produces a different effect on the flow field according to its location on the sphere body. When the synthetic jet is located at an angle of $6.5^{\circ}$ from the stagnation point we find similarities with that of the cylinder with a reduction in the wake size of the sphere and a corresponding reduction in the drag on the sphere. Changes in the flow occur upstream and downstream of the actuation point giving rise to global effects on the flow that become reduced the further away the point is from the synthetic jet.

When the synthetic jet is placed at an angle of incidence of $76^{\circ}$ the effects of localisation of the synthetic jet are amplified since the flow has almost reached the separation point. The wake region is affected more so than with the case when the synthetic jet is angled at $6.5^{\circ}$. This would suggest that less aero-shaping is occurring on the sphere surface and more energy is placed into wake modification. Although even in the wake there is more of a localised affect in the plane of the synthetic jet actuation. 
The synthetic jet is capable of improving the aerodynamic performance of 3-dimensional bluff bodies through the aero-shaping mechanism. The location of the jet closer to the stagnation point of the sphere affects the flow field globally more so than when it was located closer to the separation point since its affect was more so limited to the upper hemisphere. The synthetic jet in the wake of the sphere also improves the aerodynamic performance since the momentum of the synthetic jet is mostly transferred to the wake of sphere and does not interact with the boundary layer.

\subsection{Investigation of air jet vortex generator for active flow control 2.3.1 Background information}

The maximum normal force coefficient $\left(C_{n}\right)$ that can be generated by a single element airfoil may be limited by flow separation, which can occur at higher angles of attack. This phenomenon can often result in a sharp drop in lift coefficient $\left(\mathrm{C}_{1}\right)$, along with an associated rise in the pressure drag coefficient $\left(C_{d p}\right)$, thus; reducing the magnitude of flow separation is an attractive proposition with respect to improving the performance of an airfoil.

Flow separation appears to be a complex phenomenon that occurs due to a combination of fluid viscosity and adverse pressure gradients [24]. Adverse pressure gradients may reduce the relative motion between the various fluid particles within the boundary layer. If this relative motion is reduced to a sufficient degree, the boundary layer may separate from the surface [25].

Furnishing the boundary layer with additional momentum may allow greater penetration against adverse pressure gradients with a concomitant reduction in the magnitude of flow separation. Generating a series of longitudinal vortices over the airfoil surface appears to be one mechanism for achieving this aim [26]. This series of vortices may act in a manner such that high momentum fluid in the ambient flow field is bought down to the near wall region furnishing the boundary layer with additional momentum [27].

Longitudinal vortices can be generated by issuing small jets of air from the surface of the airfoil. The first practical application of the technique is usually attributed to Wallis [28]. Since this study, much research has been carried out on Air Jet Vortex Generators (AJVG's), where reductions in flow separation have been demonstrated under laboratory conditions on two dimensional wings undergoing cyclical [29] and non-cyclical [30] changes in angle of attack. In addition, AJVGs have successfully increased the power output of full-sized wind turbines [31]. Reducing the energy consumption required to achieve a given reduction in flow separation will further extend the utility of AJVGs as a technique for enhancing the performance of airfoils. The desirability of parameters such as the pitch and skew of the jet axis [32], as well as the orientation [33] and preference [34] for rectangular orifices appears to be relatively well established.

The key to further reductions in energy requirements may lie with studies focussing on the detailed dynamics of fluid jet behaviour. Experiments with jets issuing into quiescent bodies of fluid demonstrated the enhanced penetration of jet fluid that was either started impulsively [35], or issued in a non-steady manner with respect to time [36].

Studies conducted with fluid jets issuing into cross flows are particularly relevant to separation control applications about airfoils. Adding a non-steady characteristic to the jet injection scheme appears to allow the jet fluid to penetrate much further into a cross flow compared to a fluid jet issuing in a steady manner [37][38][39]. The exponential injection scheme of Eroglu \& Breidenthal [40] however, appears to hold the most promise in terms of a practical application as a separation control device for airfoils as the velocity profile varies with space, not time. 
The main features of the exponential jet are an injection width that increases by a given factor of " $\mathrm{e}$ " (2.71828), and a fluid injection velocity profile that also increases by the same given factor of " $\mathrm{e}$ ". The vortices generated with the device appeared to penetrate much further into the cross flow whilst also having a reduced mixing rate with the ambient fluid. A possible explanation for this behaviour suggests that the exponential parameters places high momentum jet fluid into the vortices, preventing premature weakening of this structure due to entrainment of low momentum cross flow fluid [41].

This behaviour may have interesting applications for controlling flow separation about airfoils. If the premature weakening of a vortex can be prevented, and that same vortex can effectively reduce the magnitude of flow separation, it may be possible to reduce the energy requirements associated with reducing the magnitude of flow separation.

\subsubsection{Experiment}

The exponential nozzle features an injection width and injection velocity profile that both increase by a given factor of " $\mathrm{e}$ ". An injection width and injection velocity profile that increased once by a factor of " $\mathrm{e}$ " was chosen for the present experiment. The initial injection width $\left(D_{0}\right)$ chosen was $1.5 \mathrm{~mm}$, with the total injection length along the nozzle $\left(X_{e}\right)$ set at $4 \mathrm{~mm}$. The width of the exponential nozzle thus increased from $1.5 \mathrm{~mm}$ to approximately $4.08 \mathrm{~mm}(1.5 \times \mathrm{e})$ over a distance of $4 \mathrm{~mm}$.

The exponential nozzle was discretised into four closely spaced, individual rectangular orifices (Fig. 10). The skew and pitch angles were set at 60 degrees and 30 degrees respectively, as this combination of angles produced good results in prior studies under condition of cyclical [29] and non-cyclical changes in angle of attack [30].
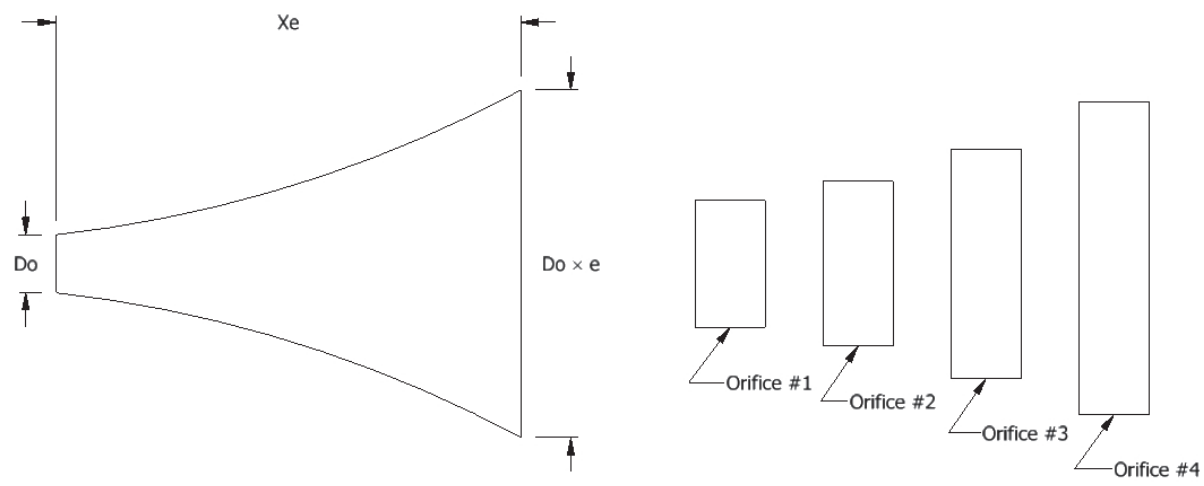

Fig. 10. Exponential nozzle \& discretised equivalent

A NACA 63-421 airfoil was equipped with an array of 24 nozzles spaced at 30mm intervals positioned at the $12.5 \%$ chord wise location. The nozzles were configured to produce a corotating series of vortices, and are similar in layout to previous studies [31][42]. The array of nozzles was designed as a homogenous structure along with the leading edge section of the airfoil and the plenum chambers supplying air to the jets (Fig. 11).

Each of the four individual rectangular orifices making up each exponential AJVG were connected to a common plenum chamber, thus; plenum chamber one was connected to, and 
supplied air to all 24 rectangular orifices labelled as \#1 (Fig 10). This arrangement was mirrored for the other three orifices, and is shown in greater detail in Fig.11.

To promote an even pressure distribution along the AJVG array, perforated brass tubes were inserted into each plenum chamber. The brass tubes were fed from both ends with pressurised air, thus minimising any static pressure variations along their length. The pressurised air was metered through conical entrance orifice plate [43][44][45] assemblies to allow measurement of the mass flow rate entering each of the four plenum chambers (Fig. 12).

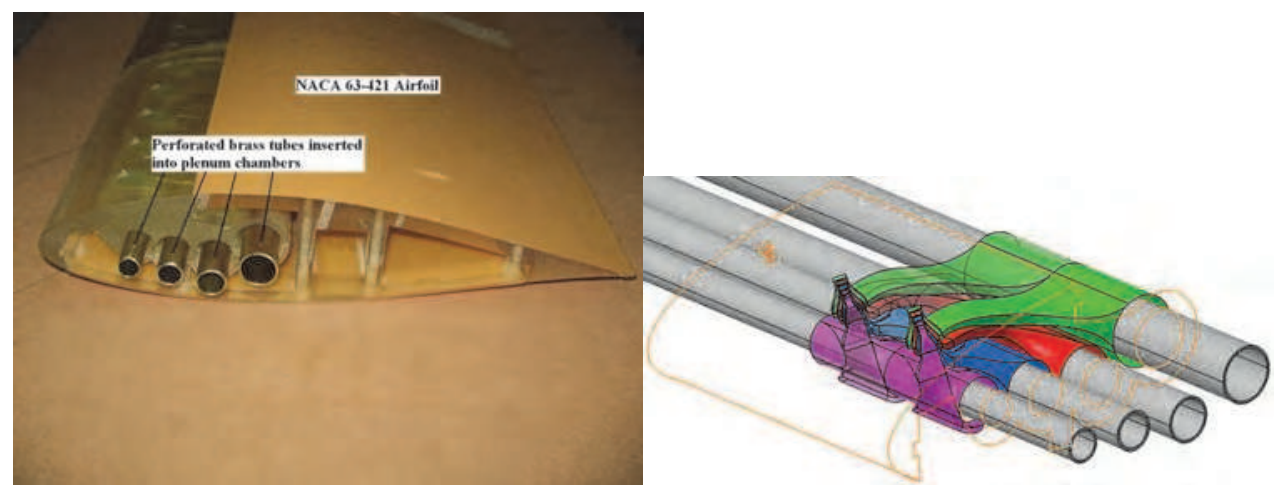

Fig. 11. Nozzle array detail

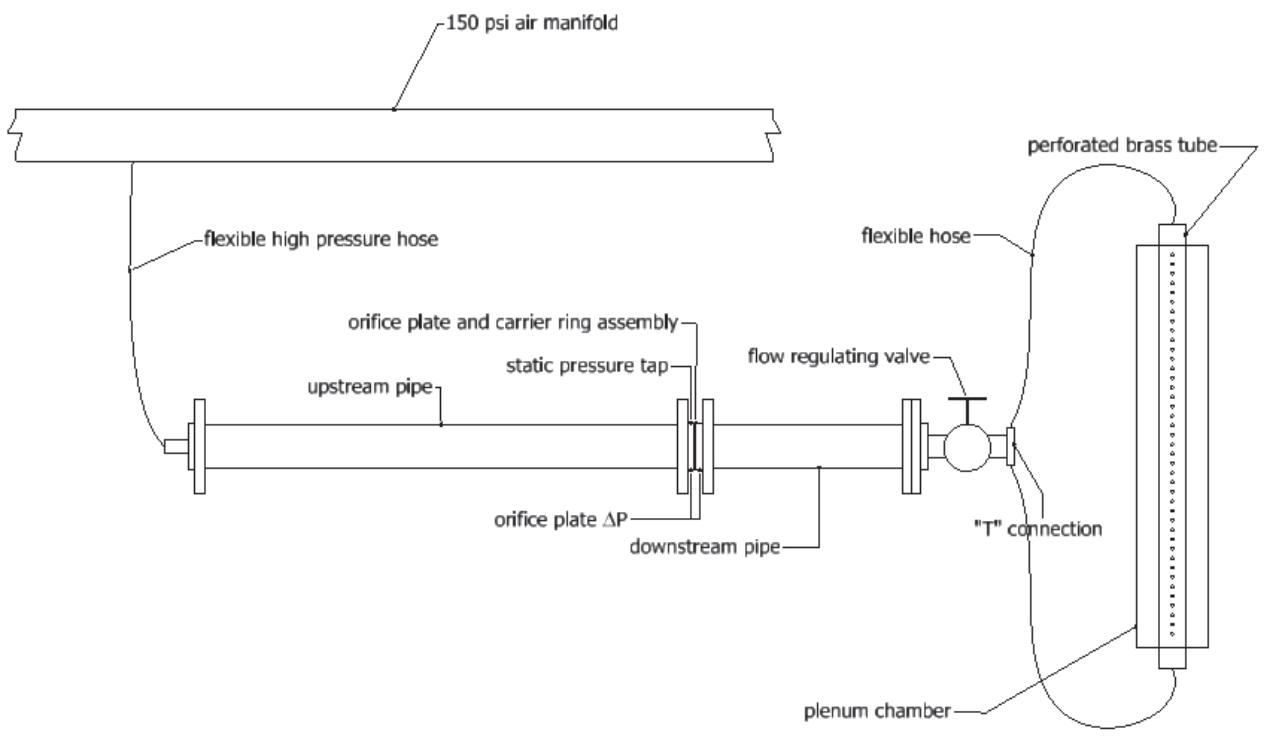

Fig. 12. Air supply schematic 
The airfoil consisted of a central section equipped with jets spanning 740mm. End plates were attached to the central section to promote two dimensional flow over the airfoil. End pieces of the same NACA 63-421 section were used to make up the full distance to the wind tunnel test section walls. The end pieces were not equipped with jets. The central part of the airfoil was constructed from Fullcure $720^{\circledR}$ polymer on an Objet Eden $260^{\circledR}$ rapid prototyping machine

Testing was conducted in the $900 \mathrm{~mm} \times 1200 \mathrm{~mm}$ test section of the large, closed circuit, subsonic wind tunnel located in the aerodynamics laboratory of the University of New South Wales. Testing was conducted at a velocity of $40 \mathrm{~m} / \mathrm{s}$, which resulted in a Reynolds number of approximately $6.4 \times 10^{5}$ based on the airfoil chord length of $250 \mathrm{~mm}$. The Reynolds number was the maximum achievable whilst keeping tunnel heating issues and errors due to blockage effects manageable. The airfoil was mounted vertically to minimise the blockage ratio, with testing conducted under conditions of free transition. The airfoil was equipped with three rows of static pressure taps, with 48 taps in each row. One row was located in the middle of the central span, with auxiliary rows $90 \mathrm{~mm}$ either side of centre. The static pressure taps were connected to a multi-tube water manometer, where the pressures taken from the centre row of taps were integrated to establish $C_{n}$ and the tangential force coefficient $\left(\mathrm{C}_{\mathrm{t}}\right)$.

The air jet injection velocities were measured using a Dantec ${ }^{\circledR}$ hotwire system. Velocity readings were taken from each of the four individual orifices making up the AJVG located nearest the centre-line of the airfoil, as well as the AJVG located on the extreme left hand side of the central airfoil section. Readings were taken at the start and finish of each test run, with all four sets of figures compensated for temperature, and averaged to establish the final velocity figures.

\subsubsection{Results and discussions}

\subsubsection{Exponential jet}

The behaviour of the vortice generated by the exponential jet is affected by the relationship between the base velocity chosen for the exponential velocity injection profile $\left(\mathrm{V}_{0}\right)$, and the velocity of the cross flow $\left(\mathrm{V}_{\infty}\right)$. Relating these two parameters to the ratio of $X_{e}$ and $D_{o}$ appeared to maximise the penetration and lifespan of the vortice [40] (Eqn. 1). For the particular orifice geometry chosen $\left(D_{0}=1.5 \mathrm{~mm}, X_{e}=4 \mathrm{~mm}\right)$, the ideal ratio between $V_{\infty}$ and $\mathrm{V}_{0}$ is 2.67 , which gives a $\mathrm{V}_{0}$ of $15 \mathrm{~m} / \mathrm{s}$ for the wind tunnel velocity of $40 \mathrm{~m} / \mathrm{s}$. This set of parameters is referred to as the "design condition" forthwith.

$$
V_{\infty}=\frac{X_{e}}{D_{o}} V_{0}
$$

Two main groups of velocity profiles were thus formulated in order to test the exponential jet. The first group featured an injection velocity that increased once by a factor of " $\mathrm{e}$ ". As with the injection width of the jet, the exponential velocity profiles were discretised into a stepwise increase in velocity (Table 1). The second group of velocity profiles had the same injection velocity across the four orifices

The mass flow rate $(\dot{m})$ entering each plenum along with the measured jet velocities ( $\mathrm{v}$ jet), dynamic pressure and wing area $\left(1 / 2 \rho v^{2} A\right)$ were combined to establish the momentum coefficient $\left(C_{\mu}\right)$, which provides an indication of the energy being consumed by the AJVG array (Eqn.2). 


$$
C \mu=\frac{2 \dot{m} v_{j e t}}{\rho v^{2} A}
$$

\begin{tabular}{|c|c|c|c|c|}
\hline $\mathrm{V}_{0}(\mathrm{~m} / \mathrm{s})$ & Orifice \#1 (m/s) & Orifice \#2 $(\mathrm{m} / \mathrm{s})$ & Orifice \#3 $(\mathrm{m} / \mathrm{s})$ & Orifice \#4 (m/s) \\
\hline 15 & 17.13 & 21.99 & 28.24 & 36.26 \\
\hline 21.6 & 24.67 & 31.67 & 40.67 & 52.22 \\
\hline 27.5 & 31.41 & 40.33 & 51.78 & 66.49 \\
\hline 32.3 & 36.89 & 47.36 & 60.82 & 78.09 \\
\hline 38 & 43.39 & 55.72 & 71.55 & 91.87 \\
\hline 43.1 & 49.22 & 63.2 & 81.15 & 104.2 \\
\hline 53.8 & 61.44 & 78.89 & 101.29 & 130.07 \\
\hline 64.6 & 73.77 & 94.72 & 121.63 & 156.18 \\
\hline
\end{tabular}

Table 1. Discretised exponential velocity profiles

$C_{1}$ is plotted as a function of angle of attack (AOA) in Fig 13. All the velocity profiles tested produced measurable gains in lift coefficient when compared to the baseline configuration with the jet array switched off. The lift curves appear to have a significant plateau region prior to the stall angle of attack, which itself appears to be affected by the operation of the jet array. The presented data has not yet been corrected for wall interference or streamline curvature, which may provide a possible explanation for this behaviour.

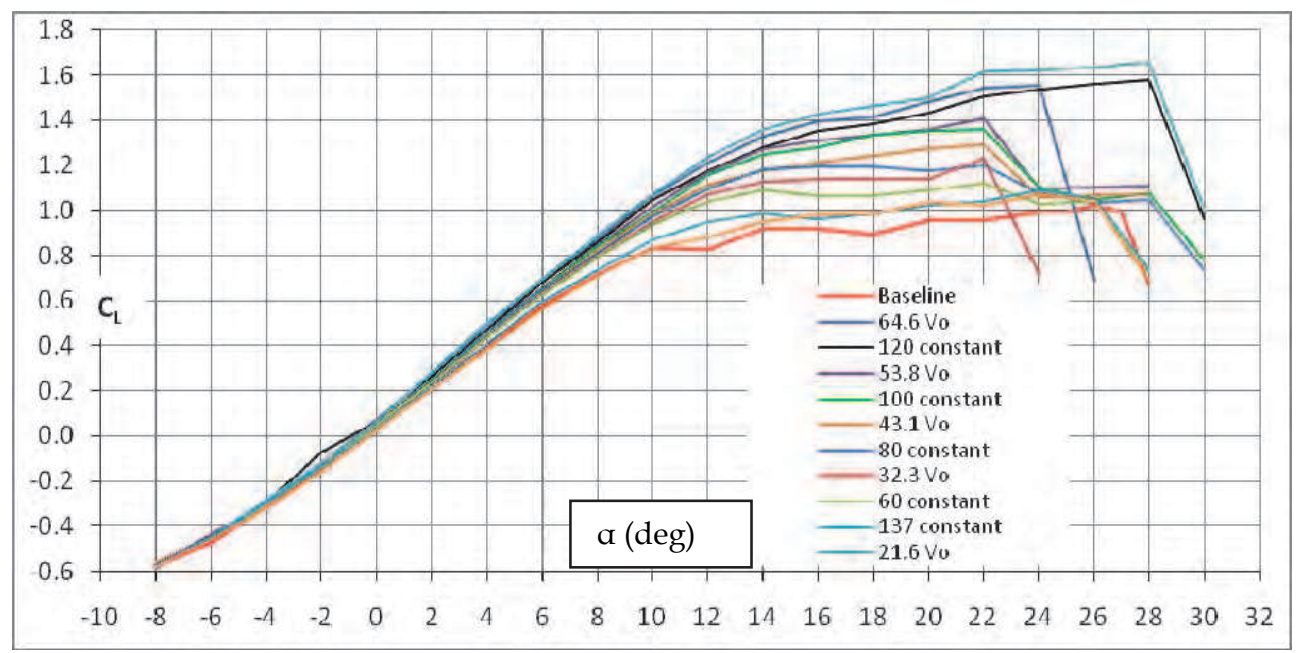

Fig. 13. Lift coefficient vs. Angle of Attack 
The average, incremental increase in $C_{1}$ over the baseline configuration (AJVG array off) was calculated for AOA's between 0-22 degrees. This range was selected as all the velocity profiles tested were able to produce positive incremental gains in $\mathrm{C}_{1}$ over this range of incidence angles. The total $C_{\mu}$ was measured at each AOA tested, with the corresponding figures for the 0-22 degrees incidence range averaged to give a final figure for comparison (Fig. 14). For average incremental increases in $C_{1}$ between $0.023-0.18$, the exponential velocity profiles $\left(V_{0}\right)$ appeared to have a lower overall $C_{\mu}$ requirement. Above this range of lift coefficients, the constant velocity profiles provided gains in $C_{1}$ for less $C_{\mu}$.

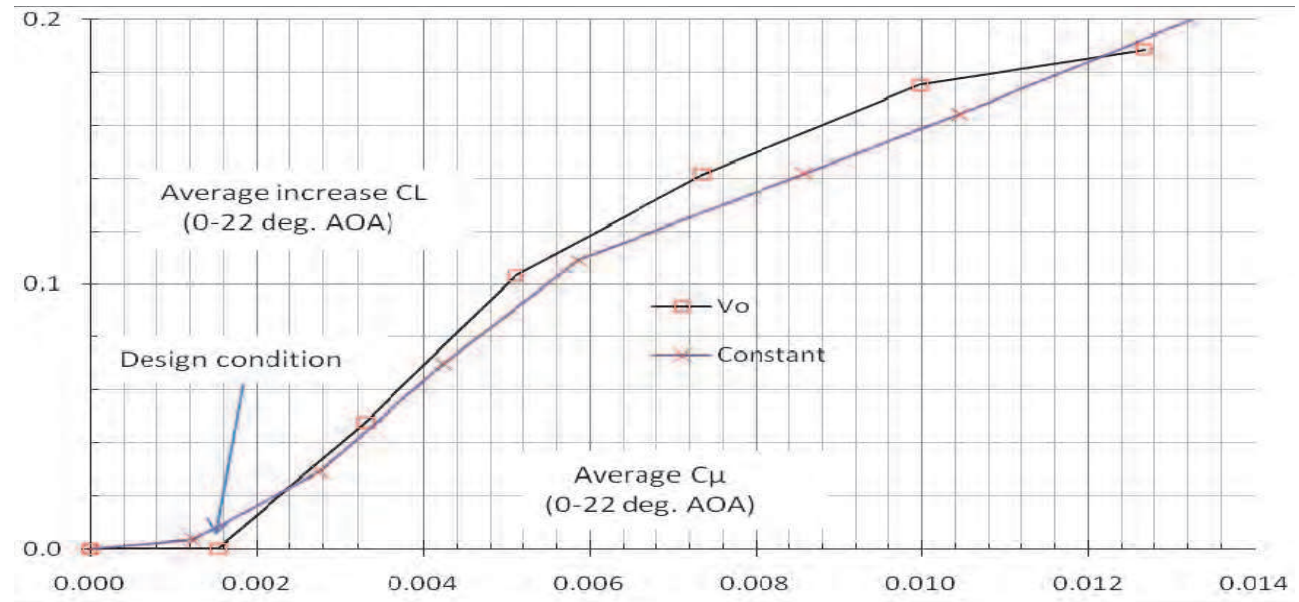

Fig. 14. Incremental increase in $\mathrm{C}_{1}$ vs. $\mathrm{C} \mu(0-22$ degrees AOA)

The greatest increase in energy efficiency appears to occur for the incremental $C_{1}$ increase of 0.16 , where the exponential jet has a $C_{\mu}$ about $12 \%$ less than that associated with using a constant injection velocity profile.

Interestingly, the exponential jet appears to provide the greatest advantages for a range of $\mathrm{C}_{\mu}$ that are somewhat beyond that associated with the design condition of the jet. The original study featured an exponential jet where the injection width and injection velocity profile both increased by a factor of " $\mathrm{e}$ " three times over a total injection length $\left(\mathrm{X}_{\mathrm{e}}\right)$ of $90 \mathrm{~mm}$. The jet was mounted on a flat plate (minimal pressure gradient) and used water as the working fluid [40]. This is in stark contrast to the present study, and may provide some possible explanations into the behaviour observed in Fig 14.

\subsubsection{Multiple orifice AJVGs}

Earlier studies conducted on AJVG's consisting of a series of closely space orifices appeared to indicate that the additional energy expended did not justify the incremental gains produced [9]. To gain a greater understanding of the phenomenon, two velocity profiles were tested at a fixed AOA (14 degrees).

The air jets issuing from the individual orifices were switched off in sequence, and the resulting change in lift coefficient calculated (Table 2). 


\begin{tabular}{|c|c|c|c|c|c|}
\hline 43.1 Vo & $\mathrm{C}_{\mathrm{L}}$ & $\Delta \mathrm{C}_{\mathrm{L}}$ & $\mathrm{C}_{\mu}$ & $\Delta \mathrm{C}_{\mu}$ & $\Delta \mathrm{C}_{\mathrm{L}} / \Delta \mathrm{C}_{\mu}$ \\
\hline All on & 1.217 & 0 & 0.012882 & 0 & 0 \\
\hline \#1 off & 1.209 & 0.008 & 0.012599 & 0.000283 & 28.268 \\
\hline \#1,2 off & 1.197 & 0.012 & 0.011621 & 0.000978 & 12.260 \\
\hline \#1,2,3 off & 1.190 & 0.007 & 0.008851 & 0.002769 & 2.527 \\
\hline All off & 0.936 & 0.254 & 0 & 0.008851 & 28.697 \\
\hline \multicolumn{7}{|c|}{} & \multicolumn{5}{|c|}{0} \\
\hline All on & 1.182 & 0 & 0.012882 & 0 & 12.089 \\
\hline \#4 off & 1.075 & 0.107 & 0.004031 & 0.008851 & 25.641 \\
\hline \#3,4 off & 1.004 & 0.071 & 0.001261 & 0.002769 & 27.579 \\
\hline \#2,3,4 off & 0.977 & 0.027 & 0.000282 & 0.000979 & 162.544 \\
\hline All off & 0.931 & 0.046 & 0 & 0.000283 & \\
\hline
\end{tabular}

\begin{tabular}{|c|c|c|c|c|c|}
\hline 80 constant & $\mathrm{C}_{\mathrm{L}}$ & $\Delta \mathrm{C}_{\mathrm{L}}$ & $\mathrm{C}_{\mu}$ & $\Delta \mathrm{C}_{\mu}$ & $\Delta \mathrm{C}_{\mathrm{L}} / \Delta \mathrm{C}_{\mu}$ \\
\hline All on & 1.201 & 0 & 0.010992 & 0 & 0 \\
\hline \#1 off & 1.168 & 0.033 & 0.009602 & 0.00139 & 23.741 \\
\hline \#1,2 off & 1.160 & 0.008 & 0.007727 & 0.001875 & 4.267 \\
\hline \#1,2,3 off & 1.143 & 0.017 & 0.005009 & 0.002718 & 6.254 \\
\hline All off & 0.925 & 0.218 & 0 & 0.005009 & 43.522 \\
\hline \multicolumn{6}{|l|}{} \\
\hline All on & 1.176 & 0 & 0.010992 & 0 & 0 \\
\hline \#4 off & 1.118 & 0.058 & 0.005983 & 0.00509 & 11.395 \\
\hline \#3.4 off & 1.069 & 0.049 & 0.003265 & 0.002718 & 18.028 \\
\hline \#2,3,4 off & 1.054 & 0.015 & 0.00139 & 0.001875 & 8.0 \\
\hline All off & 0.919 & 0.135 & 0 & 0.00139 & 97.122 \\
\hline
\end{tabular}

Table 2. Incremental changes in lift coefficient

The $43.1 \mathrm{~V}_{0}$ and 80 constant velocity profiles were chosen as they both exhibited similar behaviour in terms of $\mathrm{C}_{1}$ vs. AOA compared with the baseline configuration (Fig. 13). In both instances, measurable changes in lift coefficient were detected whenever the air supply to a plenum chamber was turned off.

$\mathrm{C} \mu$ is often added to $\mathrm{C}_{\mathrm{d}}$ to get a better picture of the overall "penalty" associated with supplying air to an AJVG array. Fig. 15 plots the ratio of $C_{l}$ and the sum of $C_{d p}$ and $C \mu$. Beyond an AOA of 12.5 degrees, it appears that $C_{1} /\left(C_{d p}+C \mu\right)$ with the jets operating is superior to $C_{1} / C_{d p}$ of the airfoil alone when the jets are switched off. 


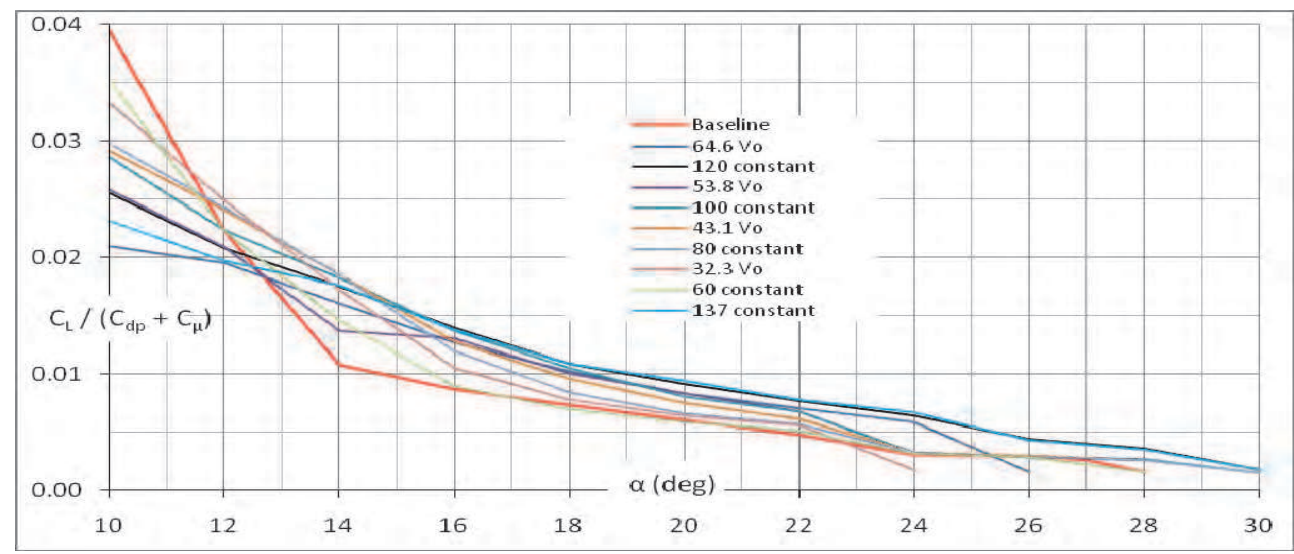

Fig. 15. $\mathrm{C}_{1}$ vs. $\left(\mathrm{C}_{\mathrm{dp}}+\mathrm{C} \mu\right)$

Taken together, the results of Fig. 14, Fig. 15 and Table 2 appear to suggest that an AJVG consisting of multiple, closely spaced orifices produces worthwhile performance gains. These performance gains may be enhanced further by using an injection width and injection velocity profile that both increase by some given factor of " $\mathrm{e}$ ".

\subsubsection{Conclusions}

An AJVG consisting of a geometrically related series of orifices was tested experimentally to determine the ability of the device to reduce the magnitude of flow separation about a NACA 63-421 airfoil. The incremental gains in $C_{1}$ were measured along with the $C \mu$ consumed by the AJVG array. For a given, average incremental increase in $\mathrm{C}_{1}$ between 0.023 - 0.18, injection velocity profiles featuring an exponential characteristic provided performance gains for less $\mathrm{C} \mu$ compared with a constant injection velocity profiles. The greatest increase in energy efficiency appeared for the incremental $C_{1}$ increase of 0.16 , where a reduction in $\mathrm{C}_{\mu}$ of about $12 \%$ was measured.

\subsection{Investigation of wind driven ventilator for performance enhancement}

Greater environmental awareness in affluent and developing countries has lead people to increasingly question the nature of progress of modern day society under pinned by technological development which in the process has also given rise to unnatural contingencies of energy utilization that have the potential to destroy the very environment which sustains life. People are, therefore, looking towards alternative energy systems that can alter the present energy use patterns that have lead to this dilemma.

One such energy system that is finding widespread use in different parts of the world is the use of natural wind as an energy source. Products such as rotating ventilators are finding use in domestic, commercial or industrial building or transport vehicles to provide optimum or at least some satisfactory environment [46], [47] in which to live or work. A rotating ventilator which is simple in structure, light in weight, cheap to install and costs nothing to operate is, therefore, proving to be an environmental friendly air extraction device. A picture of a rotating ventilator in use on the roof top of a commercial building is shown in Fig. 16. 


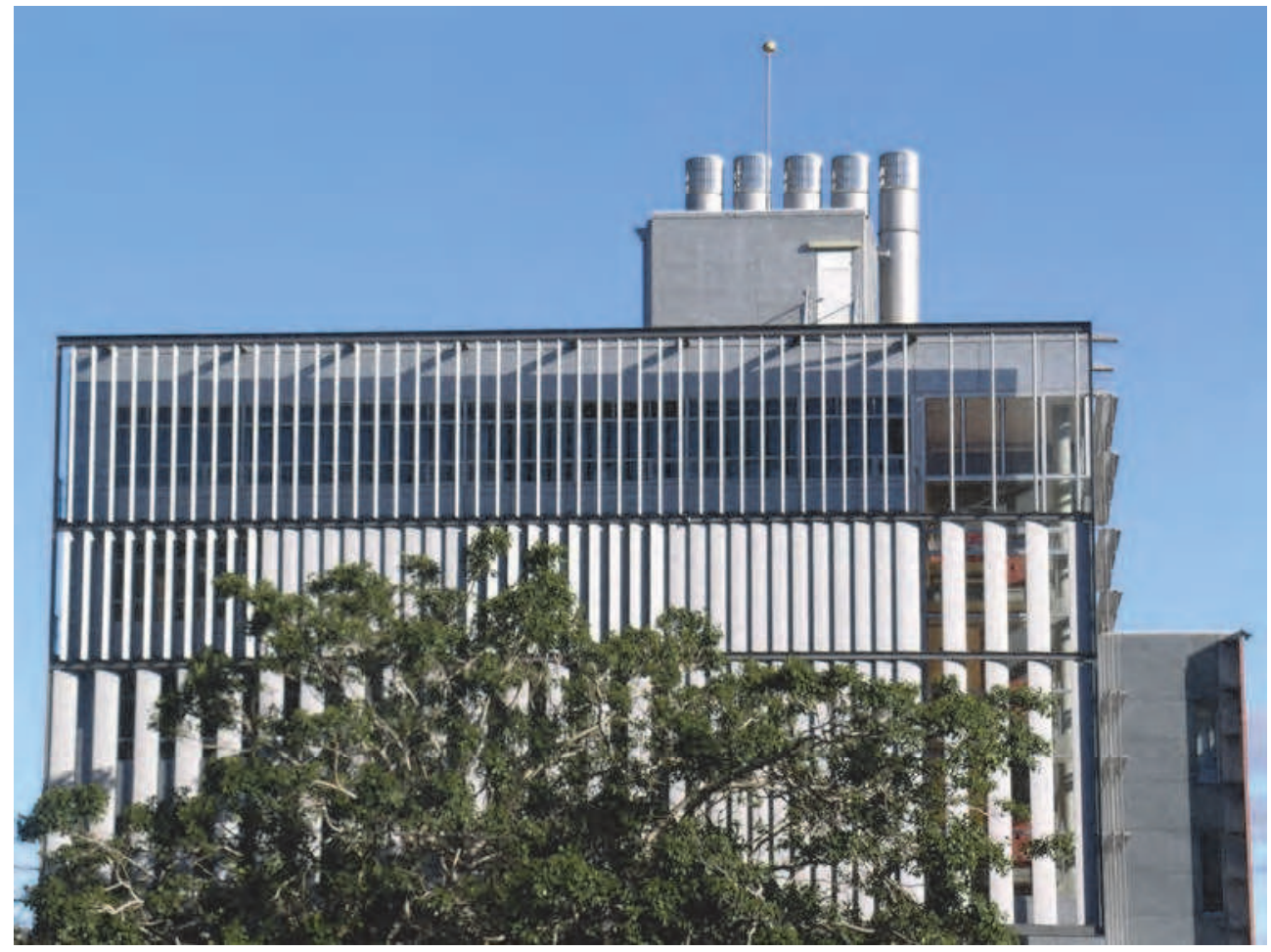

Fig. 16. Rotating ventilators in use on the roof top of building at the University of New South Wales

Generally speaking, a rotating ventilator is operated by the action of centrifugal force by creating a pressure difference, which helps expel the air out from inside a confined space. The device uses atmospheric air to rotate the ventilator head and consequently create the suction required. This suction is used to evacuate the contaminated air from the confined space. Many of these ventilators have evolved through trial and error and the flow physics associated with these ventilators is barely understood. Apart from some recent works carried out at the University of New South Wales [48], [49], [50], very little aerodynamic investigation has been carried out on the performance or operation of wind driven ventilating device. Consequently, there is a real need to improve the performance of existing roof top ventilator under various weather conditions, particularly in rain. To achieve this, a better knowledge of the aerodynamics of the flow field around the ventilator is essential. The motivation for this work is, therefore, to obtain some preliminary information of the flow to lay down the foundation of a more effective investigative wake traverse technique [51] to define performance characteristics. Although the total lift or the total drag on a ventilator can be obtained using pressure transducer of a force balance, an accurate determination of the profile and induced drag components of the total drag, however, is not easy. Recent innovative developments of techniques [2], [3], [52], [53] at the University of New South Wales for measurement in highly complex flow appear to offer the prospect of developing an effective three dimensional wake traverse technique for use in such 
situations. These considerations, therefore, prompted the formulation of the present project. Qualitative investigation of the internal flow and how it exhausts into open atmosphere is, therefore, the main objective of this study. Two differently designed rotating ventilators were used for this purpose. Some hot-wire results were also obtained to determine the mass flow extraction rate of these two ventilators.

\subsubsection{Experiment}

\subsubsection{Description of wind tunnel test facility}

The wind tunnel, Fig.17, used for the test is of the open circuit type. The wind speed is variable from 0 to $30 \mathrm{~m} / \mathrm{s}$, air being drawn at the rounded intake by an eight bladed axial flow fan with nine down stream flow straighteners. The fan is driven by a $17.5 \mathrm{kw}$ variable speed DC motor. After leaving the fan, the air stream passes through a conical angle diffuser with concentric cone flow stabilizers, one flow stabilizing screen, three flow smoothing screens and a 6:1 contraction, before discharging at the $760 \mathrm{~mm}$ diameter open test section.

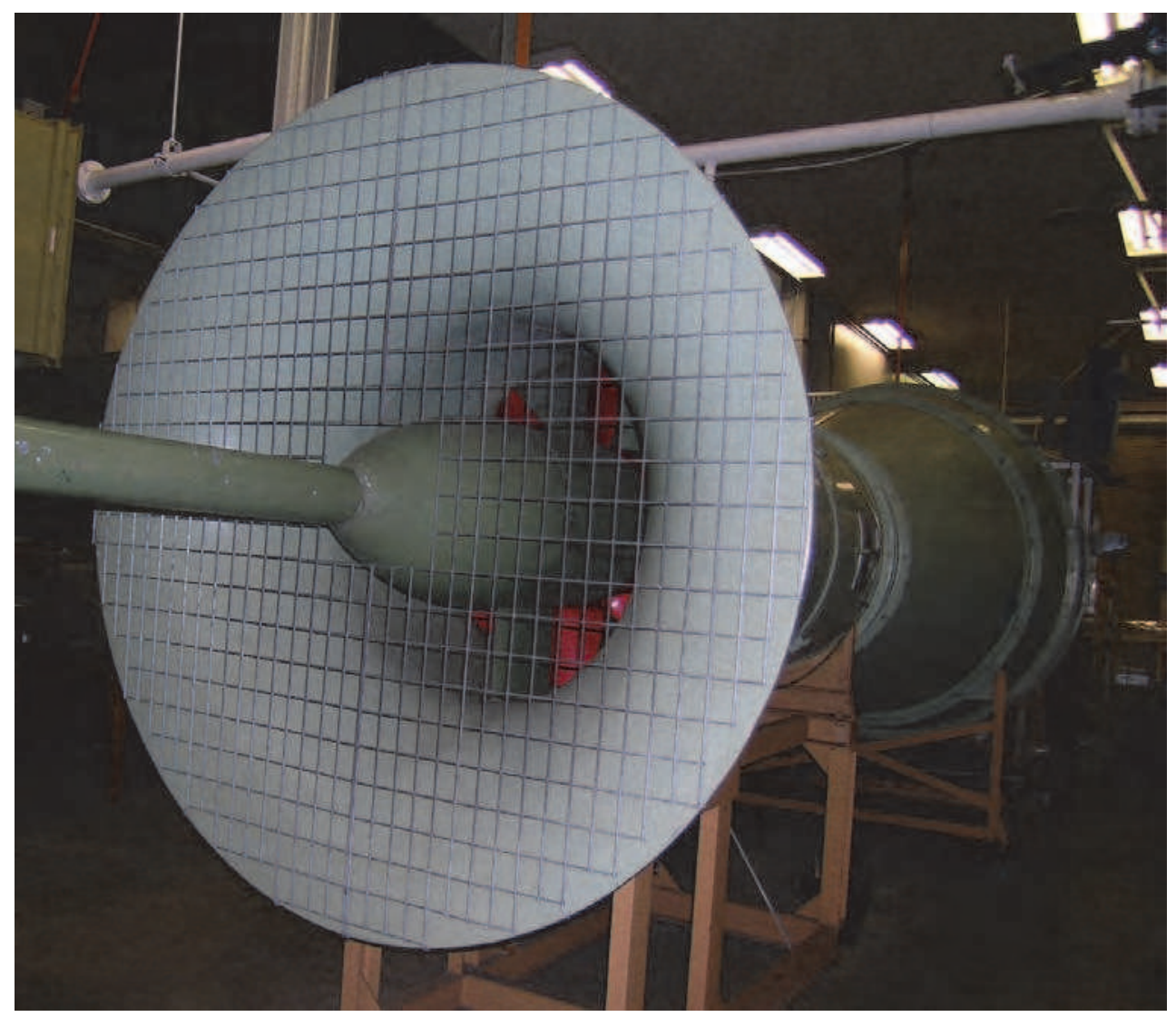

Fig. 17. Open jet wind tunnel at the University of New South Wales. 


\subsubsection{Description of test models}

Two ventilators were used in this study. For the sake of convience, they will be referred to as ventilator A and ventilator B (Figs 18a and 18b) in this chapter.

Ventilator A, Fig. 18(a), has a Savonius style 3 blade structure to drive the ventilator and an eight blade centrifugal fan to extract air. There is no air flow between the driving blades and the centrifugal fan. The overall ventilator diameter is $200 \mathrm{~mm}$, excluding the fan weather cover and the height is $120 \mathrm{~mm}$. The air inlet diameter is $100 \mathrm{~mm}$.

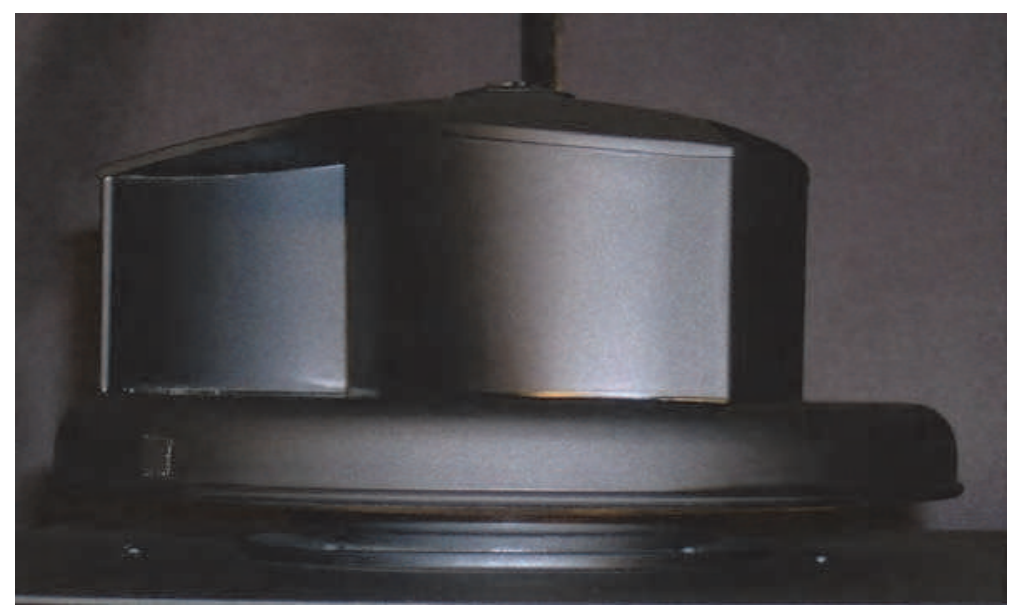

(a)

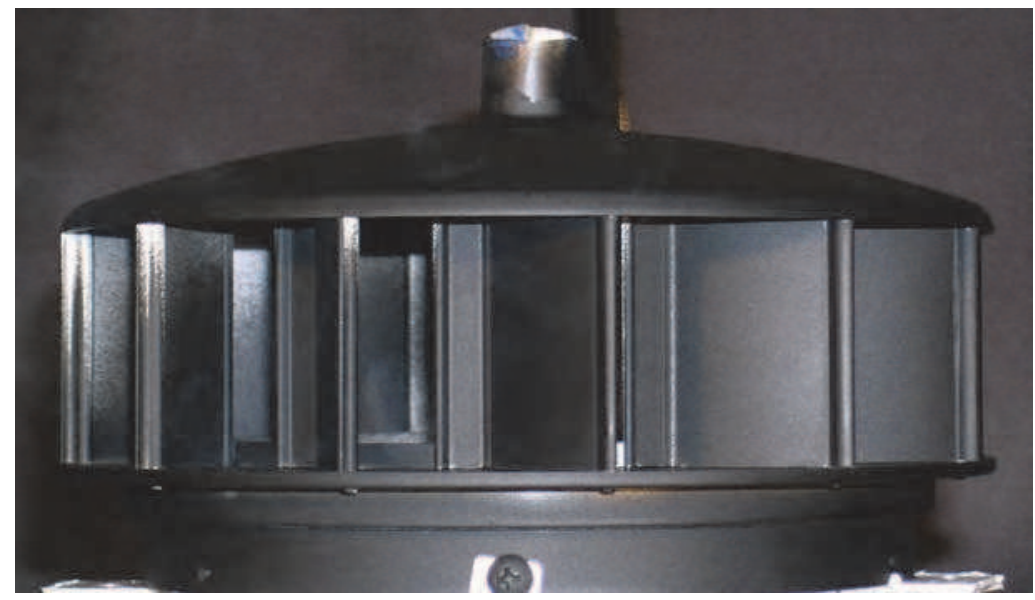

(b)

Fig. 18. (a). Ventilator A; (b). Ventilator B.

Ventilator B, Fig. 18(b), has a 12 blade centrifugal fan, which is designed to both turn the ventilator and extract air. The overall ventilator diameter is $200 \mathrm{~mm}$ and the height is $100 \mathrm{~mm}$. The air inlet diameter is $140 \mathrm{~mm}$. 


\subsubsection{Description of set up for flow visualization and flow measurement}

The arrangement of the test set up is shown in Fig. 19. The wind tunnel velocity was measured using a Pitot-static Tube, mounted at the front of the open test section and a Furness Controls FC0510 micro manometer. The inlet pipe velocity was measured by using a Dantec fibre film hotwire anemometer type $54 \mathrm{~N} 60$ located at the $3 / 4$ radius position inside a $145 \mathrm{~mm}$ diameter tube. The tube had a mitred corner with seven flow correcting vanes. Total tube length was $1620 \mathrm{~mm}$ exluding a tapered entry cone. Rotation was measured using a CDT-2000 digital tachometer using its non contact mode. Smoke was generated by a Dick Smith M6000 fog machine, located at the entry cone. The models were painted matte black, and a black back ground was used to provide contrast with the light coloured smoke. Digital photographs were taken using a Nikon Coolpix 5400 camera.

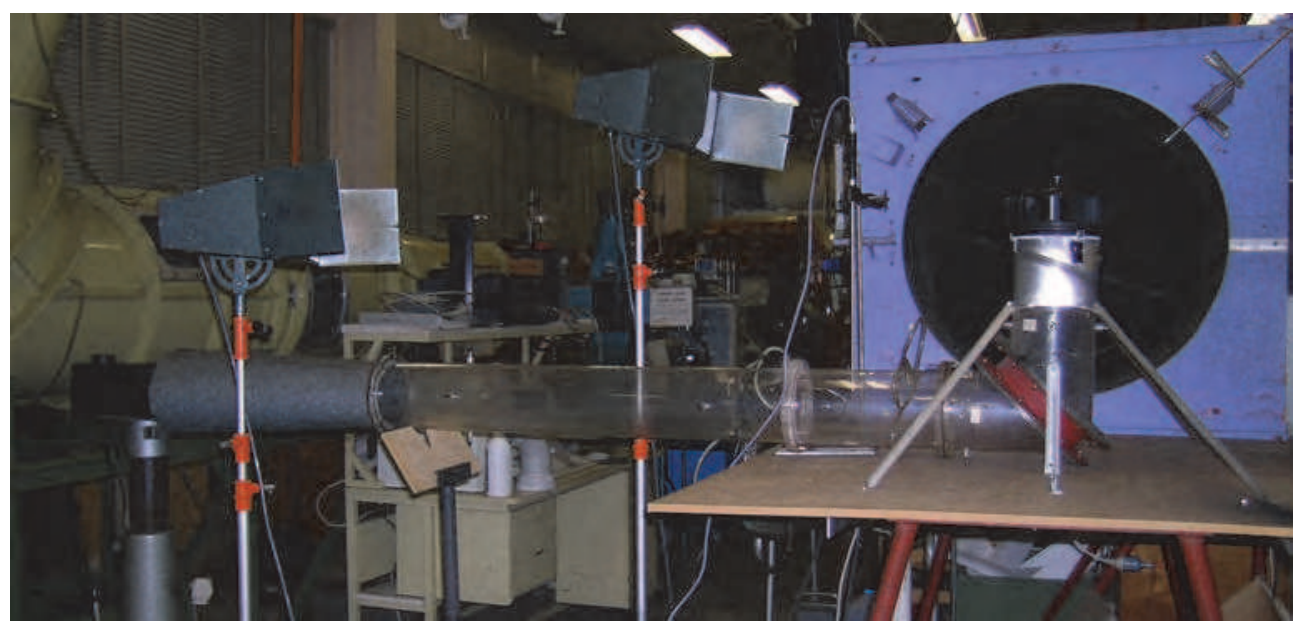

Fig. 19. Test set up

\subsubsection{Results and discussions}

\subsubsection{Qualitative: Flow visualization}

The results for exhaust flow visualisation are presented in Figs 20 and 21 and inlet flow visualization in Fig. 22.

It can be seen in Fig. 20 (a), when the wind tunnel velocity is $1 \mathrm{~m} / \mathrm{s}$, ventilator A did not rotate, the smoke exhausts from the rear half of the ventilator, and is drawn upwards and forward mixing with the air in the turbulent area behind the driving blades.

At a higher wind tunnel velocity of $9.5 \mathrm{~m} / \mathrm{s}$ ventilator A rotates at $389 \mathrm{rpm}$. The exhaust is shown to emit from the sides, front and rear of the centrifugal fan. Some of the exhaust continues be drawn up into the turbulent area behind the driving blades, Fig.20 (b).

In Fig.20 (c), the wind tunnel velocity is now $16.5 \mathrm{~m} / \mathrm{s}$ and the resultant ventilator rotation is $1619 \mathrm{rpm}$ for ventilator A. The exhaust continues from the sides, front and rear of the centrifugal turbine and to be drawn up into the turbulent area behind the driving blades.

When the wind tunnel velocity is $1 \mathrm{~m} / \mathrm{s}$ and ventilator B does not rotate, the smoke exhausts from the rear half of the ventilator, and is drawn upward. More smoke is exhausted from the camera side of the ventilator. Free stream air is drawn through the ventilator and mixes with the smoke, Fig.21 (a). 


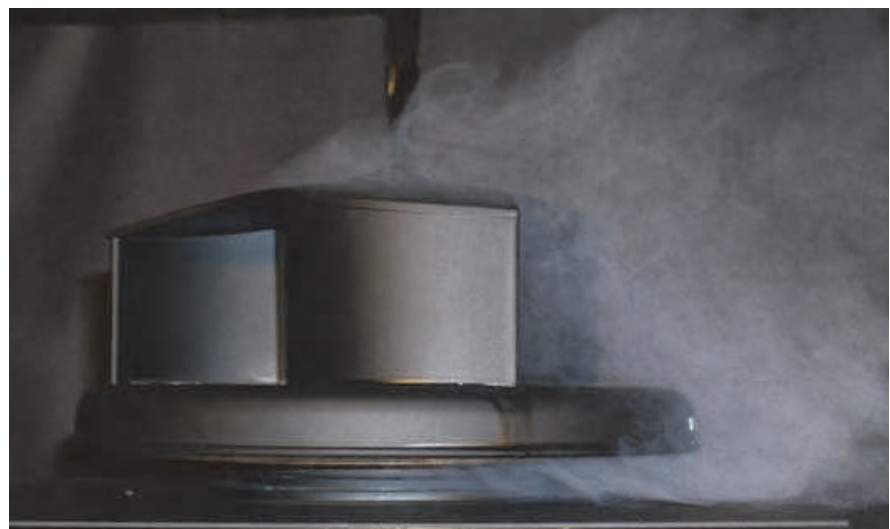

(a)

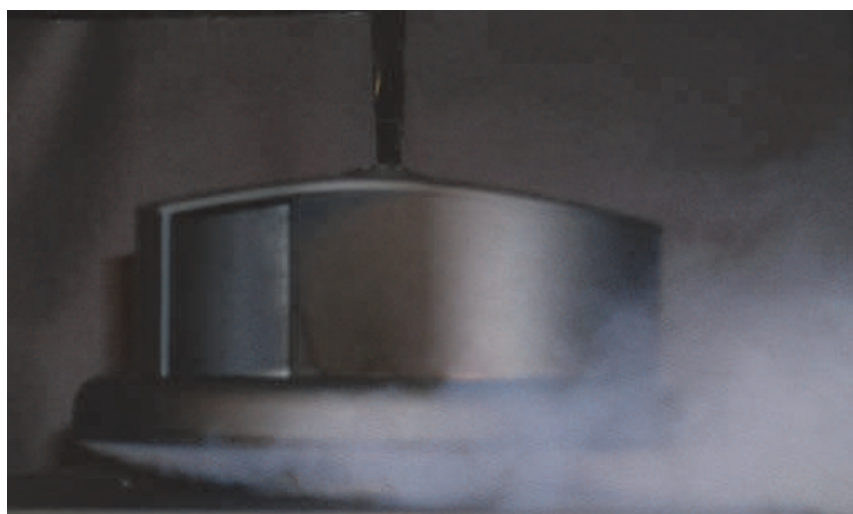

(b)

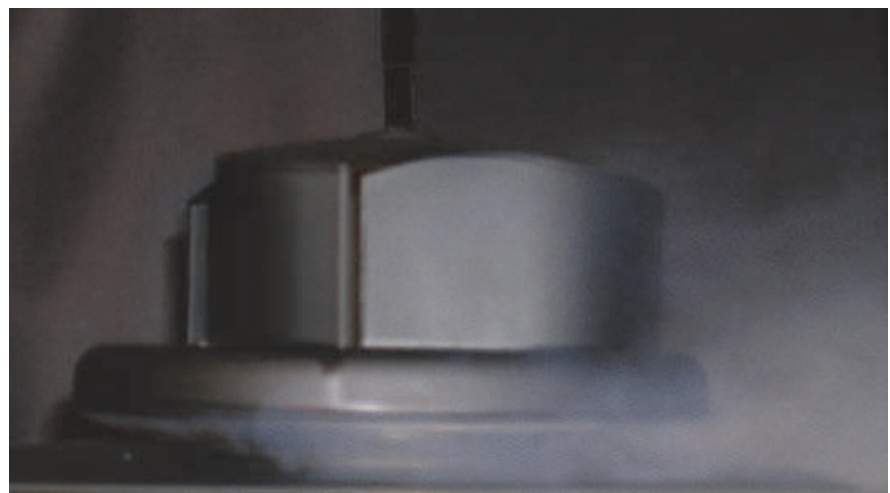

(c)

Fig. 20. Exhaust Flow visualisation using ventilator $A$ at (a) $1 \mathrm{~m} / \mathrm{s}$ and $0 \mathrm{rpm}$; (b) $9.5 \mathrm{~m} / \mathrm{s}$ and $389 \mathrm{rpm}$ and (c) $16.5 \mathrm{~m} / \mathrm{s}, 1619 \mathrm{rpm}$ 
Wind Tunnel 'Concept of Proof' Investigations

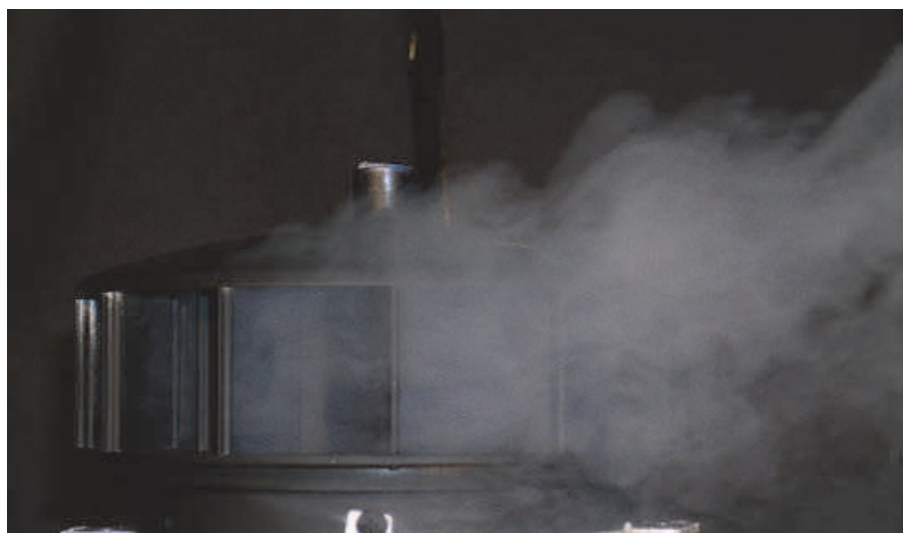

(a)

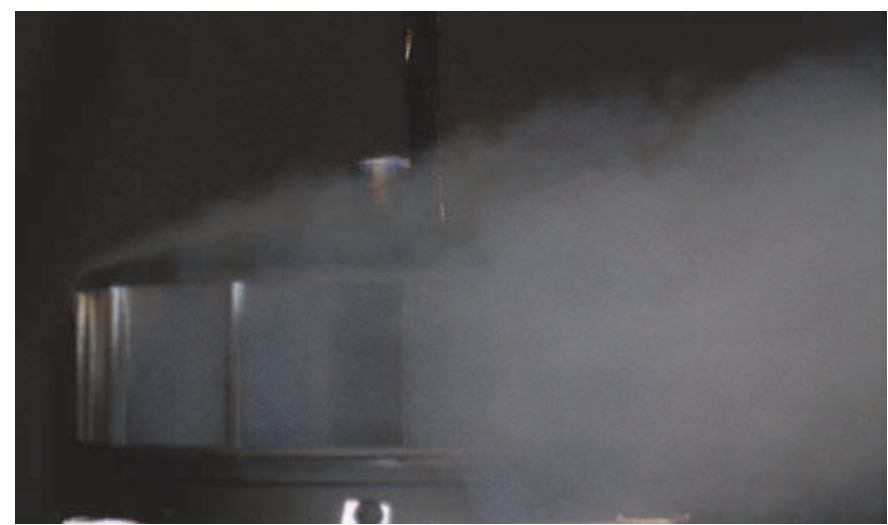

(b)

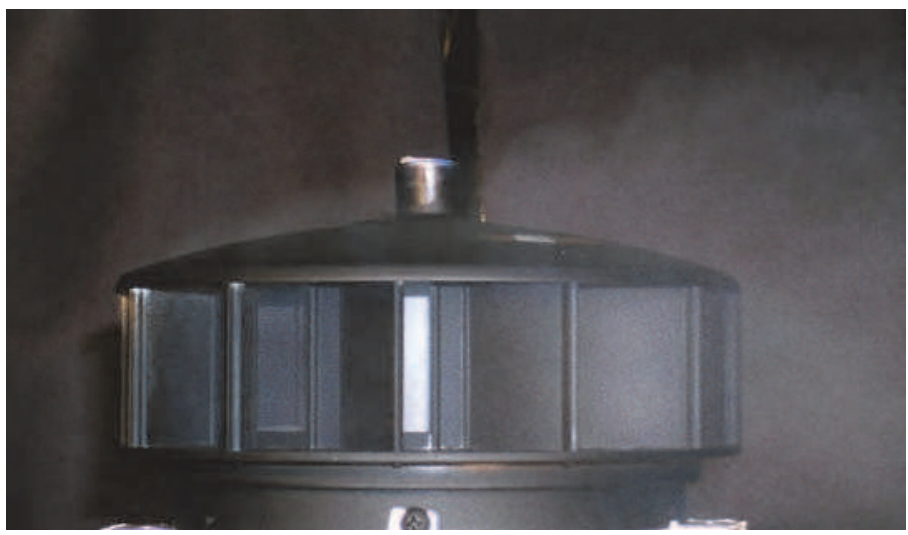

(c)

Fig. 21. Exhaust Flow visualisation using ventilator B at (a) $1 \mathrm{~m} / \mathrm{s}$ and $0 \mathrm{rpm}$; (b) $9.5 \mathrm{~m} / \mathrm{s}$ and $389 \mathrm{rpm}$ and (c) $16.5 \mathrm{~m} / \mathrm{s}, 1619 \mathrm{rpm}$ 


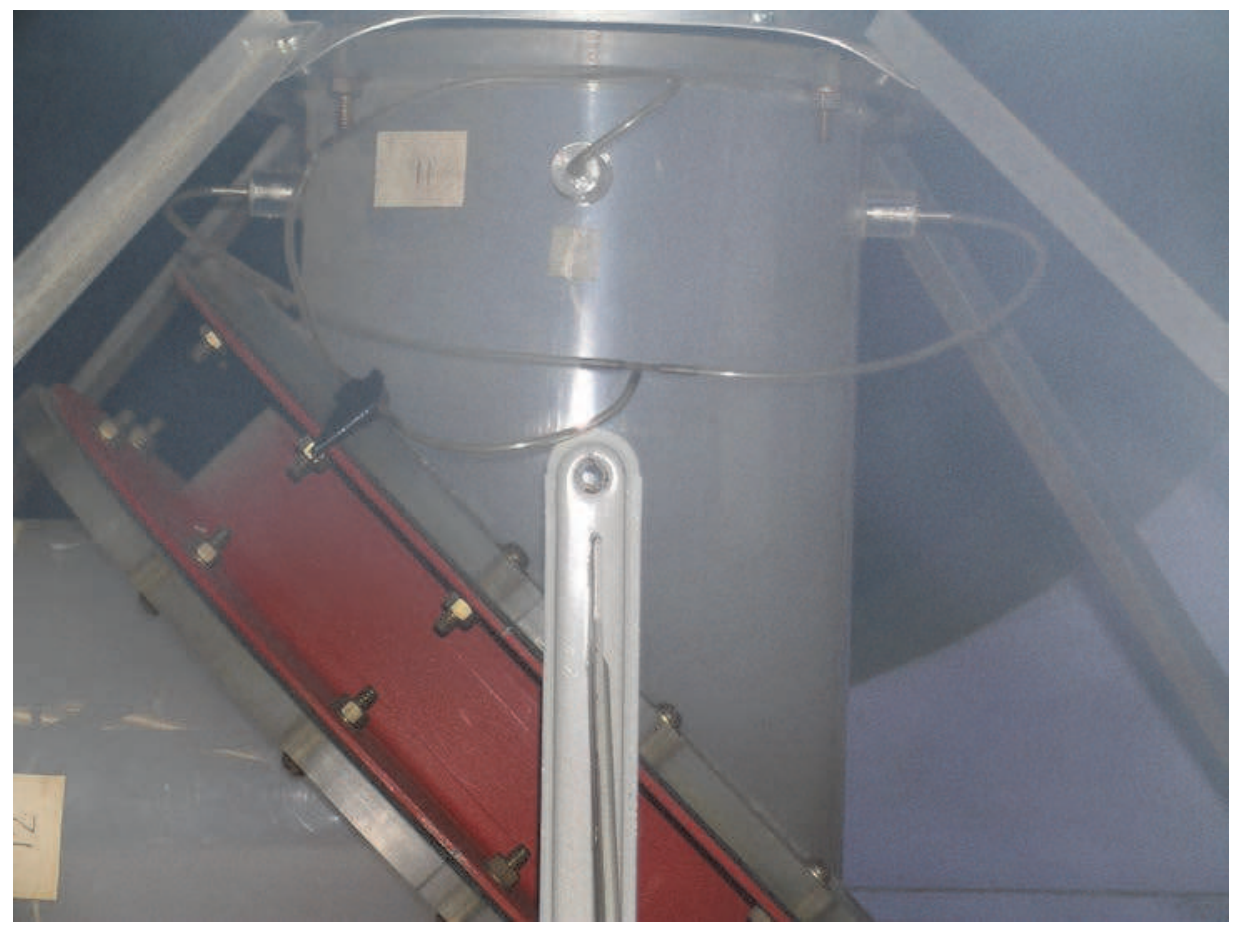

Fig. 22. Smoke flow visualisation at the intake.

When the wind tunnel velocity is $9.5 \mathrm{~m} / \mathrm{s}$ the resultant ventilator rotation is $364 \mathrm{rpm}$ for ventilator $\mathrm{B}$. The smoke is exhausted from the front, back and sides. The majority of smoke is exhausted from the rear and camera side of the ventilator. Some exhaust from the front is drawn above the ventilator, Fig 21 (b).

At the higher wind tunnel velocity of $16.5 \mathrm{~m} / \mathrm{s}$ the ventilator rotation is $674 \mathrm{rpm}$ for ventilator B. Again the smoke is exhausted from the front, back and sides and the majority of smoke is exhausted from the rear and camera side of the ventilator. Some exhaust from the front is drawn above the ventilator. Due to the higher suction, more air has mixed with the smoke before being drawn into the ventilator, making the smoke less dense, Fig. 21(c) The smoke mixed evenly inside the pipe (figure 7), making any visual interpretation of flow highly subjective. Injecting smoke into the intake tube changed the intake velocity. To confirm flow rates it was decided to use an alternative method to determine flow inside the pipe.

\subsubsection{Qualitative flow measurement}

The ventilator rotation was measured using the tachometer and the intake velocity was measured with the hot wire anemometry system for different wind tunnel speeds.

As expected from rotation comparison, Fig.23 shows a linear relationship between ventilator rotation and wind tunnel speed. A linear relationship is demonstrated between wind tunnel velocity and intake velocity as is evident from Fig.24. It is estimated that the experimental repeatability of measurements is within $\pm 1 \%$ of the measured value. 


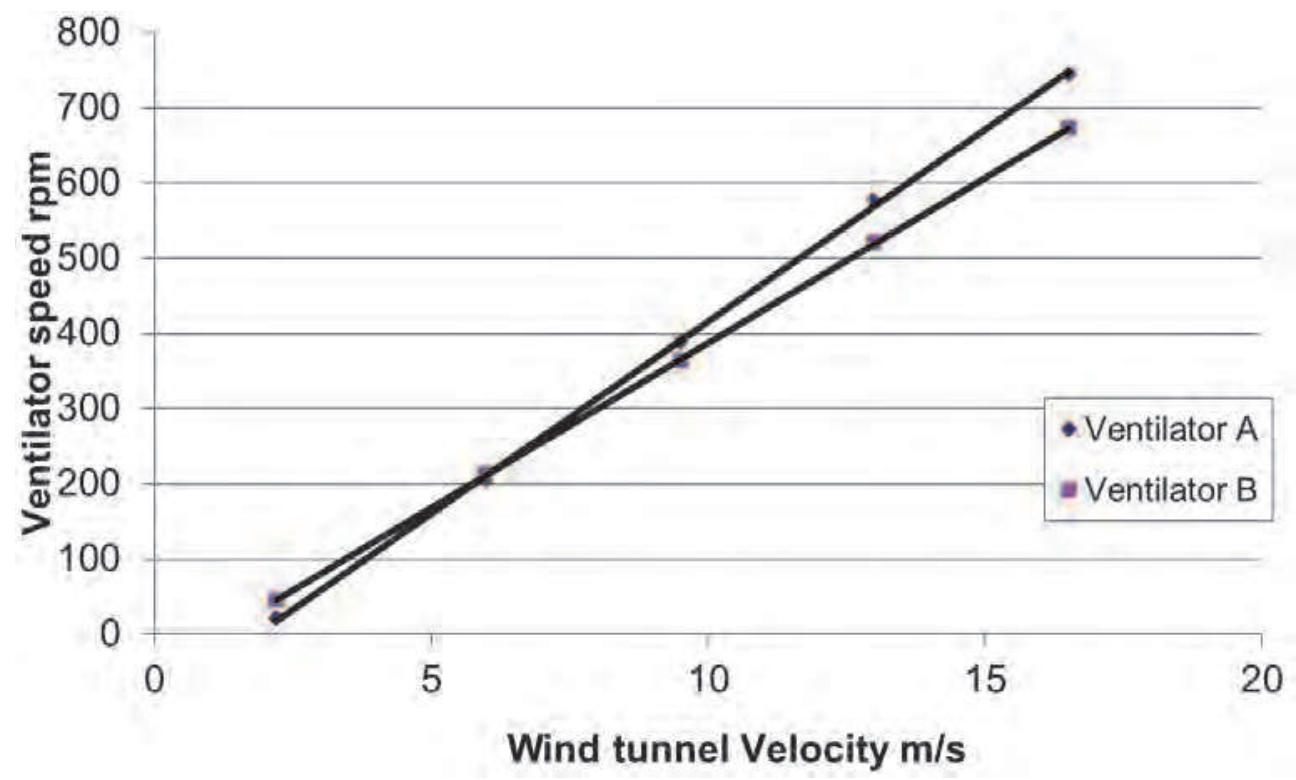

Fig. 23. Comparison of rotation speed

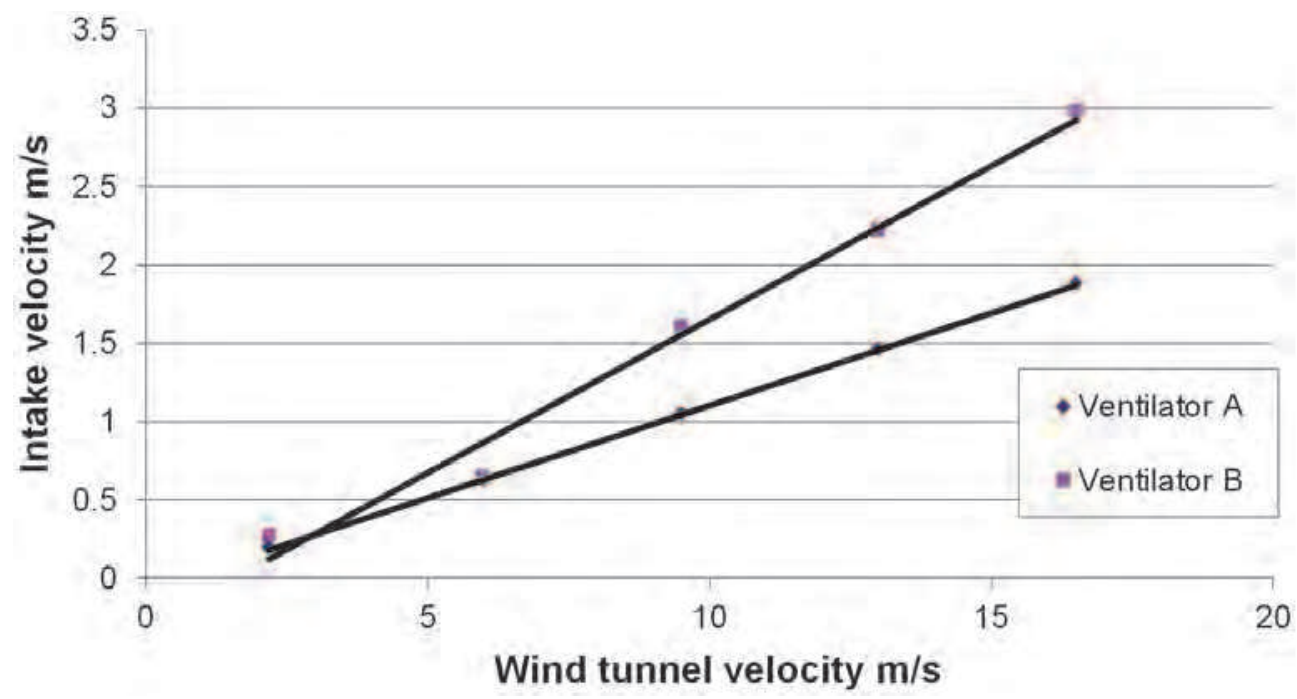

Fig. 24. Comparison of Intake velocity 


\subsubsection{Conclusions}

The complex nature of the exhaust flow is demonstrated through the use of flow visualization using smoke. In both ventilators smoke is visible at the front of the ventilator if rotating, indicating both are likely to stop water from entering during wet conditions. In terms of intake velocity flow rate, ventilator B appears better. This might be due to several factors of its design including intake diameter, blade shape, number of blades and blade area and the absence of a weather cowling. In terms of rotating speed ventilator B started at a lower wind tunnel velocity and ventilator A obtained a higher rotation speed. Again this may be due to a number of factors including blade number, shape, and torque loading.

\section{Final concluding remarks}

In this chapter, the significance of modern day usage of wind tunnels to provide 'concept of proof' validations to new and innovative ideas based primarily on the works of the authors at the University of New South Wales, have been provided. The development of a novel three-dimensional skin friction methodology has the potential to contribute to more advanced and informative investigation of fluid flow field, the studies and validation of the viability of synthetic jet or air jet vortex generator as new but powerful active flow control technologies or the studies on rotating roof ventilators have the potential to produce cost-efficient high performance products. A case in point is the subsequent works on rotating wind driven ventilators mentioned in this chapter that were conducted under the Australian Research Council Industry Collaboration Linkage Grants resulting in a more efficient blade design which has been incorporated in the 'Hurricane' ventilator with nearly $15 \%$ increase in air extraction capability at low speed and enhanced safety and performance in rain and operation. The works also lead to the basis for the concept of a hybrid ventilator [54] to overcome the dependence of conventional roof top ventilators on the availability of wind. This resulted in the 2008 AIRAH Excellence award winning hybrid ventilator, the 'ECPOWER' in the HVAC-Achiever category as a distinguished Australian product, invention or innovation in heating, ventilation and air conditioning. Both the 'Hurricane' and 'ECOPOWER' are currently marketed worldwide by CSR Edmonds Pty Ltd, Australia. Stories about the outcomes of both projects have featured in ARC reports to parliament [55].

Today wind tunnels are used quite extensively to solve aeronautical as well as nonaeronautical problems in fields as diverse as transportation, architecture or environmental concerns. With rapid advances in instrumentation and computer technologies and greater use of automated systems in experimental studies, wind tunnel is a formidable device at the disposal of engineers and scientists to advance the cause of human progress and civilization opening up possibilities of new frontier technologies and products with zero or reduced carbon footprints.

\section{Acknowledgements}

The authors would like to thank Terry Flynn, the technical officer in charge of the UNSW aerodynamics laboratory, Dr. J. Lien and Simon Shun of the UNSW for their significant assistance and contribution that has made the writing of this chapter possible. 
Acknowledgement is also made of the Australian Research Council and Allan Ramsay, Managing Director and Derek Munn of CSR Edmonds Products Pty Ltd, Australia for their financial support in carrying out many of the works mentioned in this chapter.

\section{References}

[1] A. Gatto, K.P.Byrne, N.A.Ahmed and R.D.Archer, 'Pressure Measurements over a Cylinder in Crossflow using Plastic Tubing', Experiments in Fluids, vol 30, Issue 1, pp 43-46, 2001

[2] A.Pissasale and N.A.Ahmed, 'Theoretical calibration of a five hole probe for highly three dimensional flow', International Journal of Measurement Science and Technology, vol 13, July, 2002, pp1100-1107

[3] A.Pissasale and N.A.Ahmed, "A novel method of extending the calibration range of five hole probe for highly three dimensional flows", Journal of Flow Measuremennt and Instrumentation, vol. 13, issues 1-2, March-April, 2002, pp 23-3018.

[4] T. Cebecci, and A. M. O. Smith, "Analysis of Turbulent Boundary Layers," Academic Press. (1974).

[5] A. T. Degani, F. T. Smith and J. D. A. Walker, 1993 The structure of a three-dimensional turbulent boundary layer Journal of Fluid Mechanics , 250: 43-68

[6] S.J. Lien and N. A. Ahmed, Application of multi-hole pressure probes in skin friction determination. 25th AIAA Aerodynamic Measurement Technology and Ground Testing Conference, San Francisco: Paper Number: AIAA-2006-3659 (2006)

[7] J.Lien and N.A.Ahmed, 'An examination of suitability of multi hole pressure probe technique for skin friction measurement in turbulent flow', Journal title: Flow Measurement and Instrumentation, Article accepted for publication: 30-JAN-2011

[8] J. P. Johnston, "Measurements in three-dimensional turbulent boundary layer induced by a swept, forward -facing step," J. Fluid Mech, vol. 42, part 4, 833-844 (1970)

[9] Elsenaar A and Boelsma S H 1974 Measurements of the Reynolds stress tensor in a threedimensional turbulent boundary layer under infinite swept-wing conditions National Aerospace Laboratory NRL TR 74095 U

[10] N. V. Chandrasekhara Swamy, B. H. Lakshmana Gowda, V. R. Lakshminatfi, "Measurements in 3-dimensional turbulent boundary layer on a yawed flat plate induced by leading edge vortex" Lecture notes in Physics, Springer Berlin, 217-221 (1978)

[11] J.D. Vagt, 'Experimental techniques in three-dimensional turbulent boundary layers" Proceedings of the Symposium, Berlin, West Germany, 1982 (A83-47011 22-34). Berlin, Springer-Verlag, 19-43 (1982)

[12] S. H. Chue (1975) Pressure Probes for Fluid Measurement, Prog. Aerospace Sci., Vol. 16, No. 2, pp. 147-223

[13] M.Amitay, D.R.Smith, D.E. Parekh, A.Gleizer, 'Aerodynamic flow control over an unconventional airfoil using synthetic jet actuators', AIAA .,vol. 39, No. 3, 2001, pp 361-70

[14] M.Amitay, V.Kibens, D.E. Parekh, A.Gleizer, ‘Flow reattachment dynamics over a thick airfoil controlled by synthetic jet actuators', 37th AIAA Aero. Sci. Meet., ,Reno, Nevada, 1999, pp 99-1001. 
[15] C.Y. Lee and D.B. Goldstein, 'Two-dimensional synthetic jet simulation', AIAA Fluids Meeting, 2000-0406, Denver, Colorado, 2000.

[16] S. Jeon, J. Choi, W. Jeon, H.Choi, J. Park, 'Active control of flow over a sphere for drag reduction at a subcritical Reynolds number', J. of Fluid Mech., vol. 517, 2004, pp 113129.

[17] M.Amitay and A.Gleizer, 'Synthetic Jets', Ann. Rev. of Fluid Mech., vol 34, 2002, pp503529.

[18] H.J. Kim and P.A. Durbin, 'Observations of the frequencies in a sphere wake and of drag increase by acoustic excitation', Physics of Fluids, vol.31, 1988, pp 3260-3265.

[19] R. Mittal and P. Rampunggoon, 'On the Virtual Aeroshaping Effect of Synthetic Jets', Physics of Fluids. Vol.14, No. 4, 2002, pp1533-1536.

[20] H. Schlicting, 'Boundary-Layer Theory', 6th Edn., 1968, McGraw Hill.

[21] N.A.Ahmed, and R.D.Archer, 'Post-Stall Behaviour of A Wing under Externally Imposed Sound', AIAA Journal of Aircraft, vol 38, no.5, pp961-963, SeptemberOctober, 200

[22] N.A.Ahmed and D.J.Wagner, "Vortex shedding and transition frequencies associated with flow around a circular cylinder", AIAA Journal, vol. 41, no.3, March, 2003, pp 542-544

[23] H.R. William, Jr and Pope, A., 'Low Speed Wind Tunnel Testing', 2nd ed., 1984, John Wiley and Sons

[24] P.K.Chang, 'Separation of Flow', 1970, pp.1, Pergamon Press.

[25] J.D. Anderson, J.D., 'Fundamentals of Aerodynamics', 3 ${ }^{\text {rd }}$ ed.,2001, pp. 715-717, McGraw Hill.

[26] R.A. Wallis, 'A Preliminary Note on a Modified Type of Air Jet for Boundary Layer Control',1960, pp. 2, A.R.C. /CP 513.

[27] E.L. Houghton and P.W. Carpenter, P.W., 'Aerodynamics for Engineering Students', 5th ed., 2003, pp. 513-514, Elsevier Butterworth-Heinemann.

[28] R.A. Wallis, 'The use of Air Jets for Boundary Layer Control', Aerodynamic Research Laboratories Australia, Aero Note 110, 1952.

[29] C. Singh, D.J. Peake, A. Kokkalis, V. Khodagolian, F.N. Coton and R.A.M. Galbraith, 'Control of Rotorcraft Retreating Blade Stall Using Air-Jet Vortex Generators', Journal of Aircraft, vol. 43 No. 4, 2006, pp. 1169-1176.

[30] A. Krzysiak, 'Control of Flow Separation Using Self-Supplying Air-Jet Vortex Generators', AIAA Journal, vol. 46, No. 9,2008, pp. 2229-2234.

[31] A.G. Oliver, 'Air Jet Vortex Generators for Wind turbines', PhD. dissertation, 1997, City University, London.

[32] G.V. Selby, J.C. Lin and E.G. Howard, 'Control of low-speed turbulent separated flow using jet vortex generators, Experiments in Fluid, vol. 12, No. 6, 1992pp. 394-400.

[33] A. Bridges and D.R. Smith, 'Influence of Orifice Orientation on a Synthetic Jet-Boundary Layer Interaction', AIAA Journal, vol. 41, No. 12, 2003pp. 2394-2402.

[34] X. Zhang, 'An inclined rectangular jet in a turbulent boundary layer-vortex flow', Experiments in Fluids, vol. 28, 2000, pp. 344-354.

[35] H. Johari, Q. Zhang, M.J. Rose and S.M. Bourque, 'Impulsively Started Turbulent Jets', AIAA Journal, vol. 35, No. 4, 1997, pp. 657-662. 
[36] Q. Zhang and H. Johari, 'Effects of Acceleration on Turbulent Jets', Physics of Fluids, vol 8, No. 8, 1996, pp. 2185-2195.

[37] H. Johari, M. Pacheco-Tougas and J.C. Hermanson, J, ‘Penetration and Mixing of Fully Modulated Turbulent Jets in Crossflow", AIAA Journal, vol. 37, No. 7, 1999, pp 842850 .

[38] H. Johari and G. S. Rixon, 'Effects of Pulsing on a Vortex Generator Jet', AIAA Journal, vol. 41, No. 12,2003 pp 2309-2315.

[39] A. Eroglu and R.E. Breidenthal, 'Structure, Penetration, and Mixing of Pulsed Jets in Crossflow', AIAA Journal, vol. 39, No. 3, 2001, pp 417-423.

[40] A. Eroglu and R.E. Breidenthal , 'Exponentially Accelerating Jet in Crossflow', AIAA Journal, vol. 36, No. 6, 1998, pp 1002-1009.

[41] R. Breidenthal, (1986), 'The Turbulent Exponential Jet', Physics of Fluids, vol. 29, No. 8,1986, pp. 2346-2347.

[42] T. Vronsky, 'High Performance Cost Effective Large Wind Turbine Blades Using Air jet Vortex Generators', 2000, ETSU W/41/00541/REP.

[43] ISO 5167-1:2003(E), 'Measurement of fluid flow by means of pressure differential devices inserted in circular cross-section conduits running full - Part 1: General principles and requirements'.

[44] ISO 5167-2:2003(E), 'Measurement of fluid flow by means of pressure differential devices inserted in circular cross-section conduits running full - Part 2: Orifice plates'.

[45] ISO/TR 15377:2007(E), 'Measurement of fluid flow by means of pressure differential devices - Guidelines for the specification of orifice plates, nozzles and Venturi tubes beyond the scope of ISO5167'.

[46] 'Heating Piping and Air Conditioning' Volume 47, Issue 13, December 1975, Page 5961.

[47] T.E. Kreichelt and G.R. Kern, 'Natural ventilation in hot process buildings in the steel industry', Journal of Iron and Steel Engineering, December, 1976, Page 39-46.

[48] N.A. Ahmed and J. Back, 'Destructive wind tunnel tests', UNSW Unisearch Report no. 23214-10, Australia, 1996.

[49] N.A. Ahmed and J. Back, 'Wind tunnel tests on two ventilators', UNSW Unisearch Report no. 29295-01, Australia, 1997.

[50] D.H.Rashid and N.A.Ahmed, 'Study of aerodynamic forces on a rotating wind driven ventilator', Wind Engineering, vol. 27, no.1, 2003, pp 63-72

[51] N.A. Ahmed, 'Implementation of a Momentum Integral Technique for Total Drag Determination', International Journal of Mechanical Engineering Education, vol.30, no.4, 2002, pp 62-72

[52] A.J. Pissasale and N.A.Ahmed, 'Examining the effect of flow reversal on seven-hole probe measurements', AIAA Journal, vol 41, no 12, 2003, pp 2460-2467

[53] A.J. Pissasale and N.A.Ahmed, 'Development of a functional relationship between port pressures and flow properties for the calibration and application of multi-hole probes to highly three-dimensional flows', Experiments in Fluids, March, vol 36, no.3, March, 2004, pp 422-436 
[54] S.Shun and N.A.Ahmed, 'Utilizing wind and solar energy as power sources for a hybrid building ventilation device', Renewable Energy, Volume 33, Issue 6, June 2008, Pages 1392-1397

[55] Annual Report, 2008-2009, Australian Res Council, ISSN 1444-982X, p92, (2009) 


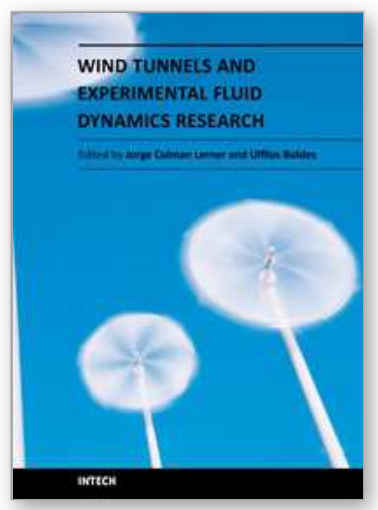

\author{
Wind Tunnels and Experimental Fluid Dynamics Research \\ Edited by Prof. Jorge Colman Lerner
}

ISBN 978-953-307-623-2

Hard cover, 709 pages

Publisher InTech

Published online 27, July, 2011

Published in print edition July, 2011

The book â€œWind Tunnels and Experimental Fluid Dynamics Researchâ€ is comprised of 33 chapters divided in five sections. The first 12 chapters discuss wind tunnel facilities and experiments in incompressible flow, while the next seven chapters deal with building dynamics, flow control and fluid mechanics. Third section of the book is dedicated to chapters discussing aerodynamic field measurements and real full scale analysis (chapters 20-22). Chapters in the last two sections deal with turbulent structure analysis (chapters 23-25) and wind tunnels in compressible flow (chapters 26-33). Contributions from a large number of international experts make this publication a highly valuable resource in wind tunnels and fluid dynamics field of research.

\title{
How to reference
}

In order to correctly reference this scholarly work, feel free to copy and paste the following:

Noor Ahmed and Nicholas Findanis (2011). Wind Tunnel 'Concept of Proof' Investigations in the Development of Novel Fluid Mechanical Methodologies and Devices, Wind Tunnels and Experimental Fluid Dynamics Research, Prof. Jorge Colman Lerner (Ed.), ISBN: 978-953-307-623-2, InTech, Available from: http://www.intechopen.com/books/wind-tunnels-and-experimental-fluid-dynamics-research/wind-tunnelconcept-of-proof-investigations-in-the-development-of-novel-fluid-mechanical-methodologi

\section{INTECH}

open science | open minds

\section{InTech Europe}

University Campus STeP Ri

Slavka Krautzeka 83/A

51000 Rijeka, Croatia

Phone: +385 (51) 770447

Fax: +385 (51) 686166

www.intechopen.com

\section{InTech China}

Unit 405, Office Block, Hotel Equatorial Shanghai

No.65, Yan An Road (West), Shanghai, 200040, China

中国上海市延安西路65号上海国际贵都大饭店办公楼 405 单元

Phone: +86-21-62489820

Fax: $+86-21-62489821$ 
(C) 2011 The Author(s). Licensee IntechOpen. This chapter is distributed under the terms of the Creative Commons Attribution-NonCommercialShareAlike-3.0 License, which permits use, distribution and reproduction for non-commercial purposes, provided the original is properly cited and derivative works building on this content are distributed under the same license. 